TRANSACTIONS OF THE

AMERICAN MATHEMATICAL SOCIETY

Volume 360, Number 7, July 2008, Pages 3875-3907

S 0002-9947(08)04443-7

Article electronically published on February 13, 2008

\title{
SEMILINEAR HYPOELLIPTIC DIFFERENTIAL OPERATORS WITH MULTIPLE CHARACTERISTICS
}

\author{
NGUYEN MINH TRI
}

\begin{abstract}
In this paper we consider the regularity of solutions of semilinear differential equations principal parts of which consist of linear polynomial operators constructed from real vector fields. Based on the study of fine properties of the principal linear parts we then obtain the regularity of solutions of the nonlinear equations. Some results obtained in this article are also new in the frame of linear theory.
\end{abstract}

\section{INTRODUCTION}

Regularity of solutions of linear differential equations has been extensively studied by many authors. In this direction many strong results were achieved. The books [3, 9, 17, 18, are good references in the area (see also the recent paper 12 and the references therein). Regularity of solutions of elliptic nonlinear differential equations and systems was studied in [1, [2], 7], 14] (see also the book [13. for more references). Regularity of classical solutions of semilinear nonelliptic differential equations was investigated in [20, and recently in [19]. In this paper we deal with a subclass of nonlinear hypoelliptic operators, namely the class of semilinear hypoelliptic operators with multiple characteristics. The paper is organized as follows. In Section 2 we recall and introduce some notation, definitions and auxiliary results. In particular, we recall the definitions of hypoellipticity with loss of $\sigma$ derivatives and of maximal $\delta$-hypoellipticity, the Hörmander condition $(\mathrm{H})_{1}$, and two theorems of Rosthchild and Stein. We then introduce the definitions of hypoellipticity for nonlinear operators, weakly maximal $\delta$-hypoellipticity, extendedly maximal hypoellipticity in a system of given real vector fields, smoothness conditions of a system of given real vector fields, and of the $(K)_{1}^{d}$ and $\left(K^{\prime}\right)_{l}^{d}$ conditions. We also define some operations on the set of multi-orders, and finally formulate without proofs some lemmas on commutators. In Section 3 we study semilinear hypoelliptic second order differential equations of Hörmander type. The main result of this section is Theorem 3.3 on hypoellipticity of the operator $\Psi_{2}$. In Section 4 we deal with semilinear hypoelliptic high order differential equations. In Subsection 4.1 we study hypoellipticity with the requirement of the condition $(\mathrm{K})_{1}^{\mathrm{d}}$. The main results in this subsection are Theorems 4.10, 4.12, 4.13, 4.22 for linear operators and Theorems 4.24, 4.25 for nonlinear operators. In Subsubsection 4.1.2 we verify the $(\mathrm{K})_{1}^{\mathrm{d}}$ condition for some particular systems of vector fields. In Subsection 4.2

Received by the editors August 15, 2006.

2000 Mathematics Subject Classification. Primary 35H10; Secondary 35A08, 35B45, 35B65.

Key words and phrases. Semilinear differential equations, a priori estimates, maximal hypoellipticity, polynomial operators constructed from real vector fields. 
we investigate hypoellipticity without the requirement of the condition $(\mathrm{K})_{1}^{\mathrm{d}}$. The main results here are Theorems 4.30, 4.33, 4.36, 4.37, 4.38, 4.40 for linear operators and Theorem 4.41 for nonlinear operators. The method we used in the paper is: based on the study of fine properties of the principal linear parts, we then obtain the regularity of solutions of the nonlinear equations. As a by-product we also obtain some new results even for linear differential operators. To be precise, let us recall and introduce some general basic notation, definitions and auxiliary results.

\section{Notation, DEFInitions AND AUXiLiary RESUlts}

Assume that $\Omega$ is a domain in $\mathbb{R}^{n}, \alpha$-multi-index: $\alpha=\left(\alpha_{1}, \ldots, \alpha_{n}\right) \in \overline{\mathbb{Z}}_{+}^{n}$, $|\alpha|=\alpha_{1}+\cdots+\alpha_{n}$, and $P(x, \partial)$ is a linear differential operator of order $m$ in $\Omega$ of the form

$$
P(x, \partial)=\sum_{|\alpha| \leqslant m} a_{\alpha}(x) \partial^{\alpha}
$$

where $a_{\alpha}(x) \in C^{\infty}(\Omega), \partial^{\alpha}=\frac{\partial^{|\alpha|}}{\partial x_{1}^{\alpha_{1}} \ldots \partial x_{n}^{\alpha_{n}}}$.

Definition 2.1. Assume that $\sigma \in \mathbb{R}$. The operator $P(x, \partial)$ is called hypoelliptic with loss of $\sigma$ derivatives in $\Omega$ if for every real number $s$ and every subdomain $\Omega^{\prime} \subset \Omega$ from $f \in \mathcal{D}^{\prime}\left(\Omega^{\prime}\right)$ and $P(x, \partial) f \in H_{\mathrm{loc}}^{s}\left(\Omega^{\prime}\right)$ it follows that $f \in H_{\mathrm{loc}}^{s+m-\sigma}\left(\Omega^{\prime}\right)$. Here $D^{\prime}\left(\Omega^{\prime}\right)$ denotes the space of distributions and $H_{\text {loc }}^{s}\left(\Omega^{\prime}\right)$ is the standard Sobolev space on $\Omega^{\prime}$.

Let $\left(x, \tau_{\alpha}\right)_{|\alpha| \leqslant m} \in \Omega \times \tilde{\Omega} \subset \mathbb{R}^{n} \times \mathbb{R}^{N}$ and $\Phi\left(x, \tau_{\alpha}\right)_{|\alpha| \leqslant m} \in C^{\infty}(\Omega \times \tilde{\Omega})$. Denote by $\Phi\left(x, \partial^{\alpha}\right)_{|\alpha| \leqslant m}$ a nonlinear differential operator in $\Omega$ of order $m$ acting as follows:

$$
\Phi\left(x, \partial^{\alpha}\right)_{|\alpha| \leqslant m}: u(x) \longrightarrow \Phi\left(x, \partial^{\alpha} u(x)\right)_{|\alpha| \leqslant m},
$$

for $u \in C^{m}(\Omega)$ and $\left(\partial^{\alpha} u\right)_{|\alpha| \leqslant m} \in \tilde{\Omega}$.

Definition 2.2. The nonlinear operator $\Phi\left(x, \partial^{\alpha}\right)_{|\alpha| \leqslant m}$ is called hypoelliptic in $\Omega$ if for every subdomain $\Omega^{\prime} \subset \Omega$ there exists a positive integer $M$ such that from $u \in C^{M}\left(\Omega^{\prime}\right)$ and $\Phi\left(x, \partial^{\alpha} u\right)_{|\alpha| \leqslant m} \in C^{\infty}\left(\Omega^{\prime}\right)$ it follows that $u \in C^{\infty}\left(\Omega^{\prime}\right)$.

We must emphasize that according to Definition 2.2 there is a subtle difference between the notation of hypoellipticity for linear and nonlinear operators. In this article we will abbreviate linear operators as operators.

Definition 2.3. A nonlinear operator $\Phi_{1}\left(x, \partial^{\alpha}\right)_{|\alpha| \leqslant m_{1}}$ is called smoothing in $\Omega$ if for every subdomain $\Omega^{\prime} \subset \Omega$ there exists a positive integer $M$ such that the mapping $\Phi_{1}\left(x, \partial^{\alpha}\right)_{|\alpha| \leqslant m_{1}}$ sends $C^{M}\left(\Omega^{\prime}\right)$ into $C^{\infty}\left(\Omega^{\prime}\right)$.

It is clear that if a nonlinear operator $\Phi_{1}\left(x, \partial^{\alpha}\right)_{|\alpha| \leqslant m_{1}}$ is smoothing in $\Omega$, then the nonlinear operator $\Phi\left(x, \partial^{\alpha}\right)_{|\alpha| \leqslant m}$ is hypoelliptic in $\Omega$, if and only if the nonlinear operator $\Phi_{1}\left(x, \partial^{\alpha}\right)_{|\alpha| \leqslant m_{1}}+\Phi\left(x, \partial^{\alpha}\right)_{|\alpha| \leqslant m}$ is hypoelliptic in $\Omega$. Assume that $f(x)$ is a function in $\Omega$. Define the operator

$$
\Phi_{1}\left(x, \partial^{\alpha}\right)_{|\alpha| \leqslant m}: u(x) \longrightarrow f(x)+\Phi\left(x, \partial^{\alpha} u(x)\right)_{|\alpha| \leqslant m} .
$$

From what we have just said it follows that if $f(x)$ is infinitely differentiable, then the operator $\Phi\left(x, \partial^{\alpha}\right)_{|\alpha| \leqslant m}$ is hypoelliptic in $\Omega$ if and only if $\Phi_{1}\left(x, \partial^{\alpha}\right)_{|\alpha| \leqslant m}$ is hypoelliptic in $\Omega$. Nonlinear hypoelliptic second order equations are discussed in 20] and [19. 
In this paper we will study a class of semilinear operators principal linear parts of which consist of polynomials constructed from real vector fields in $\Omega$. Such operators were first studied by Hörmander [10] and then subsequently by Kohn [11, Rothschild and Stein [15], Folland [6], Helffer and Nourrigat [8], .... Assume that $\left\{X_{j}\right\}_{j=1}^{k} \in T(\Omega)$, the space of real vector fields in $\Omega$, and $\iota$ is a multi-order, i.e. a sequence $\left(\iota_{1}, \ldots, \iota_{r}\right)$ with $1 \leqslant \iota_{s} \leqslant k, 1 \leqslant s \leqslant r$. We will write

$$
X^{\iota}=X_{\iota_{1}} \ldots X_{\iota_{r}}, \quad X_{\iota}=\left[X_{\iota_{1}} \ldots\left[X_{\iota_{r-1}}, X_{\iota_{r}}\right] \ldots\right]
$$

where [,] is the commutation bracket. Put $r=|\iota|$ (called the length of the multiorder $\iota$ ). For the reason of convenience we include the zero element (with zero length) into the set of multi-orders. Note that for $\iota=\left(\iota_{1}\right)$, i.e. $|\iota|=1$, we write $X_{\iota}=X_{\iota 1}$; and $X_{\iota}=0$ for $|\iota|=0$. By $\iota(j)$ we denote the number of all indexes $l$ such that $\iota_{l}=j$. In the set of multi-orders it is convenient and possible to introduce the relation of orders. Namely, assume there are given two multiorders $\iota^{1}=\left(\iota_{1}^{1}, \ldots, \iota_{r^{1}}^{1}\right)$ and $\iota^{2}=\left(\iota_{1}^{2}, \ldots, \iota_{r^{2}}^{2}\right)$. We say that $\iota^{1}$ is less than $\iota^{2}$ if $\left|\iota^{1}\right|=r^{1} \leqslant r^{2}=\left|\iota^{2}\right|$ and $\iota_{j}^{1}=\iota_{j}^{2}$ for all $j=\overline{1, r^{1}}$. If $\iota^{1}$ is less than $\iota^{2}$, then we write $\iota^{1} \preceq \iota^{2}$. If $\iota^{1} \preceq \iota^{2}$, then denote the multi-order $\iota^{3}=\left(\iota_{r^{1}+1}^{2}, \ldots, \iota_{r^{2}}^{2}\right)$ by $\iota^{2}-\iota^{1}$. We can also define the adding operation in the set of multi-orders. Namely, we call the multi-order $\iota^{3}=\left(\iota_{1}^{1}, \ldots, \iota_{r^{1}}^{1}, \iota_{1}^{2}, \ldots, \iota_{r^{2}}^{2}\right)$ the sum of $\iota^{1}$ and $\iota^{2}$ and denote it by $\iota^{1}+\iota^{2}$. Obviously, the adding operation is not commutative. We will say that $\iota^{1}$ belongs to $\iota^{2}$, and denote it by $\iota^{1} \subseteq \iota^{2}$, if $r^{1} \leqslant r^{2}$ and $\iota_{1}^{1}=$ $\iota_{1^{*}}^{2}, \ldots, \iota_{r^{1}}^{1}=\iota_{\left(r^{1}\right)^{*}}^{2} ; 1^{*}<\cdots<\left(r^{1}\right)^{*}$. In the case $\iota^{1} \subseteq \iota^{2}$ we call the multi-order $\iota^{3}=\left(\iota_{1}^{3}, \ldots, \iota_{r^{3}}^{3}\right)$ the complement of $\iota^{1}$ with respect to $\iota^{2}$, and denote it by $\iota^{2} \backslash \iota^{1}$, if $\iota^{3} \subseteq \iota^{2}$ and $\left(1^{* *}, \ldots,\left(r^{3}\right)^{* *}\right)=\left(1, \ldots, r^{2}\right) \backslash\left(1^{*}, \ldots,\left(r^{1}\right)^{*}\right)$. Now let us recall the following condition of Hörmander:

Condition $(\mathrm{H})_{1}$ : there is a natural number $l$ such that commutators $\left\{X_{\iota}\right\}_{|\iota| \leqslant l}$ span the whole space $\mathbb{R}^{n}$ at every point in $\Omega$.

It is clear that if $l_{1} \geq l_{2}$, then the condition $(\mathrm{H})_{l_{2}}$ is stronger than the condition $(\mathrm{H})_{l_{1}}$. The condition $(\mathrm{H})_{1}$ means that the system $\left\{X_{j}\right\}_{j=1}^{k}$ is elliptic in $\Omega$. As is standard, by $(,)_{s},\|\cdot\|_{s}$ we denote the scalar product and norm in the Sobolev space $H^{s}$. In [15] Rothschild and Stein established the following a priori estimates, which will be frequently used later on in this paper.

Theorem 2.4. Assume that the vector fields $\left\{X_{j}\right\}_{j=1}^{k}$ satisfy the condition $(\mathrm{H})_{1}$ in $\Omega$. Then for every $s \in \mathbb{R}$ and any compact $K$ in $\Omega$ there exists a constant $C$ such that

$$
\begin{array}{r}
\|f\|_{s+\frac{1}{l}} \leqslant C\left(\sum_{j=1}^{k}\left\|X_{j} f\right\|_{s}+\|f\|_{s}\right), \\
\sum_{j=1}^{k}\left\|X_{j} f\right\|_{s+\frac{1}{\tau}} \leqslant C\left(\sum_{j=1}^{k}\left\|X_{j}^{2} f\right\|_{s}+\|f\|_{s}\right), \\
\sum_{i, j=1}^{k}\left\|X_{i} X_{j} f\right\|_{s} \leqslant C\left(\sum_{j=1}^{k}\left\|X_{j}^{2} f\right\|_{s}+\|f\|_{s}\right)
\end{array}
$$

for all $f \in C_{0}^{\infty}(K)$.

Note that in Theorem 2.4 the number $\frac{1}{l}$ is the best possible value. In [10] Hörmander proved a little weaker a priori estimate than in [15]. For a fixed system 
of vector fields $\left\{X_{j}\right\}_{j=1}^{k}$, in their work [8], Helffer and Nourrigat consider operators of the form

$$
Q_{m}:=Q_{m}(x, \partial)=\sum_{|\iota| \leqslant m} a_{\iota}(x) X^{\iota},
$$

where $a_{\iota}(x) \in C^{\infty}(\Omega)$ (in what follows it is convenient to assume that for $\iota=0$ the notation $X^{\iota}$ stands for the unit operator). They introduced the following definition of maximal $\delta$-hypoellipticity.

Definition 2.5. Let $\delta \in(0,1]$. We say that $Q_{m}(x, \partial)$ is a maximally $\delta$-hypoelliptic operator in $\Omega$, if for any compact $K$ in $\Omega$ there exists a constant $C$ such that

$$
\sum_{|\iota| \leqslant m}\left\|X^{\iota} f\right\|_{\delta(m-|\iota|)} \leqslant C\left(\left\|Q_{m}(x, \partial) f\right\|_{0}+\|f\|_{0}\right)
$$

for all $f \in C_{0}^{\infty}(K)$.

Definition 2.6. Let $\delta \in(0,1]$. We call $Q_{m}(x, \partial)$ a weakly maximally $\delta$-hypoelliptic operator in $\Omega$, if for any compact $K$ in $\Omega$ there exists a constant $C$ such that

$$
\sum_{|\iota| \leqslant m-1}\left\|X^{\iota} f\right\|_{\delta(m-|\iota|)} \leqslant C\left(\left\|Q_{m}(x, \partial) f\right\|_{0}+\|f\|_{0}\right)
$$

for all $f \in C_{0}^{\infty}(K)$.

Remark 2.7. If $Q_{m}(x, \partial)$ is a maximally $\delta$-hypoelliptic operator in $\Omega$, then it is hypoelliptic with loss of $m(1-\delta)$ derivatives in $\Omega$. The maximally $\delta$-hypoelliptic operator $Q_{m}(x, \partial)$ is elliptic if and only if $\delta=1$.

Remark 2.8. Let the vector fields $\left\{X_{j}\right\}_{j=1}^{k}$ satisfy the condition $(\mathrm{H})_{1}$ in $\Omega$. Then $Q_{m}(x, \partial)$ is maximally $\frac{1}{l}$-hypoelliptic in $\Omega$ if and only if $Q_{m}(x, \partial)+L(x, \partial)$ is maximally $\frac{1}{l}$-hypoelliptic in $\Omega$ for any differential operator $L(x, \partial)$ having the form

$$
L(x, \partial)=\sum_{|\iota| \leqslant m-1} b_{\iota}(x) X^{\iota} .
$$

Indeed, we only need to prove the necessary condition since the other way is obvious. Let $K$ be any compact in $\Omega$. By induction on $i$ with the use of Theorem 2.4, we have

$$
\sum_{|\iota|=i}\left\|X^{\iota} f\right\|_{s} \leqslant C\left(\sum_{|\iota|=i+1}\left\|X^{\iota} f\right\|_{s-\frac{1}{l}}+\|f\|_{s}\right) \leqslant \varepsilon \sum_{|\iota|=i+1}\left\|X^{\iota} f\right\|_{s}+C(\varepsilon)\|f\|_{s}
$$

for all $s \in \mathbb{R}, i \in \overline{\mathbb{Z}}_{+}, \varepsilon>0$ and $f \in C_{0}^{\infty}(K)$. Hence

$$
\begin{aligned}
\left\|\left(Q_{m}(x, \partial)+L(x, \partial)\right) f\right\|_{0} & \geq\left\|Q_{m}(x, \partial) f\right\|_{0}-\|L(x, \partial) f\|_{0} \\
& \geq C_{1} \sum_{|\iota|=m}\left\|X^{\iota} f\right\|_{0}-\varepsilon \sum_{|\iota|=m}\left\|X^{\iota} f\right\|_{0}-C(\varepsilon)\|f\|_{0} .
\end{aligned}
$$

The last inequality with $\varepsilon=\frac{C_{1}}{2}$ gives the estimate (2.2) for all highest derivatives in the definition of maximal $\delta$-hypoellipticity. Using the just obtained inequality together with the condition $(\mathrm{H})_{1}$ it is not difficult to get the needed estimates for

$$
\sum_{|\iota|=m-1}\left\|X^{\iota} f\right\|_{\frac{1}{\tau}}, \sum_{|\iota|=m-2}\left\|X^{\iota} f\right\|_{\frac{2}{l}}, \ldots,\|f\|_{\frac{m}{l}} .
$$


Now let us recall the notion of weighted Sobolev spaces associated with the vector fields $\left\{X_{j}\right\}_{j=1}^{k}$, which was first introduced by Rothschild and Stein in [15].

Definition 2.9. Let $1 \leqslant p \leqslant \infty$. For every nonnegative integer $m$ we denote by $S_{p, \text { loc }}^{m,\left\{X_{j}\right\}_{j=1}^{k}}(\Omega)$ the set of functions $f$ such that for any compact $K$ in $\Omega$ the following inequality holds: $\sum_{|\iota| \leqslant m}\left\|X^{\iota} f\right\|_{L^{p}(K)}<\infty$. Put $S_{p, \text { loc }}^{\infty,\left\{X_{j}\right\}_{j=1}^{k}}(\Omega)=$ $\bigcap_{m=0}^{\infty} S_{p, \text { loc }}^{m,\left\{X_{j}\right\}_{j=1}^{k}}(\Omega)$. The spaces $S_{2, \text { loc }}^{m,\left\{X_{j}\right\}_{j=1}^{k}}(\Omega)$ and $S_{2, \text { loc }}^{\infty,\left\{X_{j}\right\}_{j=1}^{k}}(\Omega)$ will simply be denoted by $S_{\mathrm{loc}}^{m,\left\{X_{j}\right\}_{j=1}^{k}}(\Omega)$ and $S_{\mathrm{loc}}^{\infty,\left\{X_{j}\right\}_{j=1}^{k}}(\Omega)$, respectively.

Definition 2.10. We will say that the system of vector fields $\left\{X_{j}\right\}_{j=1}^{k}$ satisfies the smoothness condition, if there exists a map $T: \overline{\mathbb{Z}}_{+} \longrightarrow \mathbb{R}$, with $\lim _{m \rightarrow+\infty} T(m)=$ $+\infty$, such that $S_{\mathrm{loc}}^{m,\left\{X_{j}\right\}_{j=1}^{k}}(\Omega) \subset H_{\mathrm{loc}}^{T(m)}(\Omega)$ for any $m \in \overline{\mathbb{Z}}_{+}$.

It is easy to check that the space $S_{\mathrm{loc}}^{m,\left\{X_{j}\right\}_{j=1}^{k}}(\Omega)$ enjoys the following properties:

a) $C^{m}(\Omega) \subset S_{\text {loc }}^{m,\left\{X_{j}\right\}_{j=1}^{k}}(\Omega), \forall m \geq 0$.

b) If $f \in S_{\mathrm{loc}}^{m,\left\{X_{j}\right\}_{j=1}^{k}}(\Omega)$ and $|\iota| \leqslant m$, then $X^{\iota} f \in S_{\mathrm{loc}}^{m-|\iota|,\left\{X_{j}\right\}_{j=1}^{k}}(\Omega)$.

c) If $m \leqslant \tilde{m}$, then $S_{\mathrm{loc}}^{\tilde{m},\left\{X_{j}\right\}_{j=1}^{k}}(\Omega) \subset S_{\mathrm{loc}}^{m,\left\{X_{j}\right\}_{j=1}^{k}}(\Omega)$.

d) If the vector fields $\left\{X_{j}\right\}_{j=1}^{k}$ satisfy the condition $(\mathrm{H})_{1}$, then they also satisfy the smoothness condition since $S_{\mathrm{loc}}^{m,\left\{X_{j}\right\}_{j=1}^{k}}(\Omega) \subset H_{\mathrm{loc}}^{\frac{m}{l}}(\Omega)$ (see [15]).

e) If the vector fields $\left\{X_{j}\right\}_{j=1}^{k}$ satisfy the smoothness condition, then

$$
S_{\mathrm{loc}}^{\infty,\left\{X_{j}\right\}_{j=1}^{k}}(\Omega)=C^{\infty}(\Omega) .
$$

Indeed, the inclusion

$$
S_{\mathrm{loc}}^{\infty,\left\{X_{j}\right\}_{j=1}^{k}}(\Omega)=\bigcap_{m=0}^{\infty} S_{\mathrm{loc}}^{m,\left\{X_{j}\right\}_{j=1}^{k}}(\Omega) \subset \bigcap_{m=0}^{\infty} H_{\mathrm{loc}}^{T(m)}(\Omega)=H_{\mathrm{loc}}^{\infty}(\Omega)=C^{\infty}(\Omega)
$$

holds. The converse is obvious.

Together with the linear operator $Q_{m}(x, \partial)$ in (2.1) we will as well consider its semilinear perturbation

$$
\Theta_{m}:=\Theta_{m}\left(x, \partial^{\alpha}\right): f \longrightarrow Q_{m}(x, \partial) f+\Phi\left(x, X^{\iota} f\right)_{|\iota| \leqslant m-1},
$$

where $\Phi\left(x, \tau_{\iota}\right)_{|\iota| \leqslant m-1}$ is a smooth function.

Definition 2.11. The operator $Q_{m}(x, \partial)$ is called extendedly maximally hypoelliptic in the system $\left\{X_{j}\right\}_{j=1}^{k}$ in domain $\Omega$, if for any natural number $m_{1} \in \overline{\mathbb{Z}}_{+}$from $f \in L_{\mathrm{loc}}^{2}(\Omega), Q_{m}(x, \partial) f \in S_{\mathrm{loc}}^{m_{1},\left\{X_{j}\right\}_{j=1}^{k}}(\Omega)$ it follows that $f \in S_{\mathrm{loc}}^{m_{1}+m,\left\{X_{j}\right\}_{j=1}^{k}}(\Omega)$.

Definition 2.12. The nonlinear operator $\Theta_{m}\left(x, \partial^{\alpha}\right)$ is called extendedly maximally hypoelliptic in the system $\left\{X_{j}\right\}_{j=1}^{k}$ in $\Omega$, if there exists a nonnegative integer $M$ such that for any natural number $m_{1} \in \overline{\mathbb{Z}}_{+}$from $f \in C^{M}(\Omega), \Theta_{m} f \in S_{\mathrm{loc}}^{m_{1},\left\{X_{j}\right\}_{j=1}^{k}}(\Omega)$ it follows that $f \in S_{\mathrm{loc}}^{m_{1}+m,\left\{X_{j}\right\}_{j=1}^{k}}(\Omega)$. 
Remark 2.13. If the operator $Q_{m}\left(\Theta_{m}\right)$ is extendedly maximally hypoelliptic in the system $\left\{X_{j}\right\}_{j=1}^{k}$, satisfying the smoothness condition, then it is hypoelliptic.

Next we give some lemmas on commutators, which will be used frequently in the paper. We omit the proofs of these lemmas which can easily be executed by induction.

Lemma 2.14. Let $A, B$ be elements of an algebra $\mathcal{A}$. The following formulas hold:

$$
\begin{aligned}
& A^{n} B=B A^{n}+\sum_{i=1}^{n} C_{n}^{i}\left[{ }_{i} A, B\right] A^{n-i}=\sum_{i=0}^{n} C_{n}^{i}\left[{ }_{i} A, B\right] A^{n-i}, \\
& B A^{n}=A^{n} B+\sum_{i=1}^{n} C_{n}^{i} A^{n-i}\left[B,{ }_{i} A\right]=\sum_{i=0}^{n} C_{n}^{i} A^{n-i}\left[B,{ }_{i} A\right],
\end{aligned}
$$

in which we have used the following notation:

$$
\begin{aligned}
& \left[{ }_{0} A, B\right]=B,\left[{ }_{i} A, B\right]=\underbrace{[A \ldots[}_{i \text { times }} A, B] \ldots], \text { for } i \geq 1 \text {; } \\
& {\left[B,{ }_{0} A\right]=B,\left[B,{ }_{i} A\right]=[\ldots[B, A] \underbrace{\ldots A}_{i \text { times }}], \text { for } i \geq 1 \text {. }}
\end{aligned}
$$

Lemma 2.15. Let $A, B$ be elements of an algebra $\mathcal{A}$. Then we have

$$
\begin{aligned}
A^{n} B^{m} & =\sum_{i_{1}=0}^{n} \sum_{i_{2}=0}^{n-i_{1}} \cdots \sum_{i_{m}=0}^{n-i_{1}-\cdots-i_{m-1}} \frac{n !}{i_{1} ! \cdots i_{m} !}\left[{ }_{i_{1}} A, B\right] \cdots\left[i_{m} A, B\right] A^{n-i_{1}-\cdots-i_{m}}, \\
B^{m} A^{n} & =\sum_{i_{1}=0}^{n} \sum_{i_{2}=0}^{n-i_{1}} \cdots \sum_{i_{m}=0}^{n-i_{1}-\cdots-i_{m-1}} \frac{n !}{i_{1} ! \cdots i_{m} !} A^{n-i_{1}-\cdots-i_{m}}\left[B, i_{m} A\right] \cdots\left[B, i_{1} A\right] .
\end{aligned}
$$

Lemma 2.16. Let $A_{1}, \ldots, A_{n}, B$ be elements of an algebra $\mathcal{A}$ and $A^{\alpha}=A_{1}^{\alpha_{1}} \ldots A_{n}^{\alpha_{n}}$, where $\alpha=\left(\alpha_{1}, \ldots, \alpha_{n}\right) \in \overline{\mathbb{Z}}_{+}^{n}$. The following formulas hold:

$$
\begin{aligned}
A^{\alpha} B & =\sum_{i \leqslant \alpha} C_{\alpha}^{i}\left[i_{1} A_{1} \ldots\left[{ }_{i_{n}} A_{n}, B\right] \ldots\right] A^{\alpha-i}:=\sum_{i \leqslant \alpha} C_{\alpha}^{i}\left[{ }_{i} A, B\right] A^{\alpha-i}, \\
B A^{\alpha} & =\sum_{i \leqslant \alpha} C_{\alpha}^{i} A^{\alpha-i}\left[\ldots\left[B,{ }_{i_{n}} A_{n}\right] \ldots i_{1} A_{1}\right]:=\sum_{i \leqslant \alpha} C_{\alpha}^{i} A^{\alpha-i}\left[B,{ }_{i} A\right],
\end{aligned}
$$

where the following notations are used:

$$
i=\left(i_{1}, \ldots, i_{n}\right) \in \overline{\mathbb{Z}}_{+}^{n}, C_{\alpha}^{i}=\frac{\prod_{j=1}^{n} \alpha_{j} !}{\prod_{j=1}^{n} i_{j} !\left(\alpha_{j}-i_{j}\right) !}, A^{\alpha-i}=A_{1}^{\alpha_{1}-i_{1}} \ldots A_{n}^{\alpha_{n}-i_{n}} .
$$

Lemma 2.17. Let $A$ be an operator acting on a space of functions. Then we have

$$
\begin{aligned}
& X^{\iota} A=A X^{\iota}+\sum_{\iota^{1} \subseteq \iota ; \iota^{1} \neq \iota}\left[\iota \iota^{1} X, A\right] X^{\iota^{1}}, \\
& A X^{\iota}=X^{\iota} A+\sum_{\iota^{1} \subseteq \iota ; \iota^{1} \neq \iota} X^{\iota^{1}}\left[A, \iota \backslash \iota^{1} X\right],
\end{aligned}
$$

in which $\left[{ }_{\vartheta} X, A\right]=\left[X_{\vartheta_{1}}\left[\ldots\left[X_{\vartheta_{r}}, A\right]\right]\right]$ and $\left[A,{ }_{\vartheta} X\right]=\left[\left[\left[A, X_{\vartheta_{1}}\right] \ldots\right] X_{\vartheta_{r}}\right]$ for arbitrary multi-orders $\vartheta=\left(\vartheta_{1}, \ldots, \vartheta_{r}\right)$. 
Lemma 2.18. The following formulas hold:

$$
X^{\iota^{1}} X^{\iota^{2}}=X^{\iota^{2}} X^{\iota^{1}}+\sum_{\substack{\iota^{3}|+| \iota^{4}|=| \iota^{1}|+| \iota^{2}|| \iota^{3} \mid \geq 2}} C\left(\iota^{3}, \iota^{4}\right) X_{\iota^{3}} X^{\iota^{4}}
$$

in which $C\left(\iota^{3}, \iota^{4}\right)$ are just constants.

We conclude this section by introducing two conditions, which will be used later on:

Condition $(\mathrm{K})_{1}^{\mathrm{d}}$ : for every $i \leqslant d$ and any compact $K$ in $\Omega$ there exists a constant $C=C(l, K)$ such that

$$
\left\|X_{\iota} f\right\|_{s} \leqslant C\left(\sum_{j=1}^{k}\left\|X_{j} f\right\|_{s+\frac{i-1}{l}}+\|f\|_{s}\right), \quad \forall|\iota|=i, f \in C_{0}^{\infty}(K) .
$$

Obviously, if $l_{1} \geq l_{2}$, then the condition $(\mathrm{K})_{\mathrm{l}_{2}}^{\mathrm{d}}$ is stronger than the condition $(\mathrm{K})_{\mathrm{l}_{1}}^{\mathrm{d}}$. If $d_{1} \geq d_{2}$, then the condition $(\mathrm{K})_{1}^{\mathrm{d}_{1}}$ is stronger than the condition $(\mathrm{K})_{1}^{\mathrm{d}_{2}}$. If the vector fields $\left\{X_{j}\right\}_{j=1}^{k}$ satisfy the condition $(\mathrm{K})_{1}^{\mathrm{d}}$ for all $d$, then we say that they satisfy the condition $(\mathrm{K})_{1}$.

Condition $\left(\mathrm{K}^{\prime}\right)_{1}^{\mathrm{d}}$ : for every $i \leqslant d$ and any compact $K$ in $\Omega$ there exists a constant $C=C(l, K)$ such that

$$
\left\|X_{\iota} f\right\|_{s} \leqslant C\left(\sum_{j}^{k}\left\|X_{j}^{i-1} f\right\|_{s+\frac{1}{l}}+\|f\|_{s}\right), \quad \forall|\iota|=i, f \in C_{0}^{\infty}(K) .
$$

If the condition $(\mathrm{H})_{1}$ is satisfied, then the condition $\left(\mathrm{K}^{\prime}\right)_{1}^{\mathrm{d}}$ becomes weaker than the condition $(\mathrm{K})_{1}^{\mathrm{d}}$.

\section{Semilinear hypoelliptic SECOND ORDER DifFERENTIAL EQUATIONS OF HÖRMANDER TYPE}

In this section we consider the following semilinear differential equation of Hörmander type:

$$
\Psi_{2}\left(x, \partial^{\alpha}\right) f:=P_{2}(x, \partial) f+\Phi\left(x, f, X_{1} f, \ldots, X_{k} f\right)=0,
$$

where $P_{2}(x, \partial)=\sum_{j=1}^{k} X_{j}^{2}$. Note that in view of Theorem 2.4 the operator $P_{2}$ is maximally $\frac{1}{l}$-hypoelliptic if the vector fields $\left\{X_{j}\right\}_{j=1}^{k}$ satisfy the condition $(\mathrm{H})_{1}$ in $\Omega$. For studying the nonlinear operator $\Psi_{2}\left(x, \partial^{\alpha}\right)$ we will use the following theorem, which is also due to Rothschild and Stein [15.

Theorem 3.1. Assume that the vector fields $\left\{X_{j}\right\}_{j=1}^{k}$ satisfy the condition $(\mathrm{H})_{1}$ in $\Omega$. If $f \in \mathcal{D}^{\prime}(\Omega), P_{2} f \in S_{\mathrm{loc}}^{m,\left\{X_{j}\right\}_{j=1}^{k}}(\Omega)$, then $f \in S_{\mathrm{loc}}^{m+2,\left\{X_{j}\right\}_{j=1}^{k}}(\Omega)$ for each nonnegative integer $m$. In other words, the operator $P_{2}$ is extendedly maximally hypoelliptic in $\Omega$.

Proposition 3.2. Assume that the vector fields $\left\{X_{j}\right\}_{j=1}^{k}$ satisfy the condition $(\mathrm{H})_{1}$ in $\Omega$ and $m>n l+q$. If $\Phi\left(x, \tau_{\vartheta}\right)_{|\vartheta| \leqslant q} \in C^{\infty}$ and $f \in S_{\mathrm{loc}}^{m,\left\{X_{j}\right\}_{j=1}^{k}}(\Omega)$, then $\Phi\left(x, X^{\vartheta} f\right)_{|\vartheta| \leqslant q} \in S_{\text {loc }}^{m-q,\left\{X_{j}\right\}_{j=1}^{k}}(\Omega)$. 
Proof. It suffices to prove that $X^{\iota} \Phi\left(x, X^{\vartheta} f\right)_{|\vartheta| \leqslant q} \in L_{\text {loc }}^{2}(\Omega)$ for any multi-order $\iota$ such that $|\iota| \leqslant m-q$. Since $m>n l+q$, it follows that $X^{\vartheta} f \in C(\Omega)$ for all multiorders $\vartheta$ such that $|\vartheta| \leqslant q$. Furthermore by induction on $|\iota|$ it is easy to check that $X^{\iota} \Phi\left(x, X^{\vartheta} f\right)_{|\vartheta| \leqslant q}$ is a linear combination of terms of the form

$$
a_{\alpha}(x) \frac{\partial^{\alpha} \Phi\left(x, X^{\vartheta} f\right)_{|\vartheta| \leqslant q}}{\partial x^{\alpha}}
$$

and

$$
a_{\alpha, \beta}(x) \frac{\partial^{\alpha+\beta} \Phi\left(x, X^{\vartheta} f\right)_{|\vartheta| \leqslant q}}{\partial x^{\alpha} \partial \tau^{\beta}}\left(X^{\iota^{1}} X^{\vartheta^{1}} f\right)^{m_{1}} \cdots\left(X^{\iota^{p}} X^{\vartheta^{p}} f\right)^{m_{p}},
$$

where $\alpha=\left(\alpha_{1}, \ldots, \alpha_{n}\right), \quad \beta=\left(\beta_{\vartheta}\right)_{|\vartheta| \leqslant q}$ - multi-indexes; $\tau=\left(\tau_{\vartheta}\right)_{|\vartheta| \leqslant q} ;\left|\iota^{1}\right|, \ldots,\left|\iota^{p}\right|$, $m_{1}, \ldots, m_{p} \geq 1,\left|\iota^{1}\right| m_{1}+\cdots+\left|\iota^{p}\right| m_{p} \leqslant m-q ;\left|\vartheta^{j}\right| \leqslant q ; a_{\alpha}(x), a_{\alpha, \beta}(x) \in C^{\infty}(\Omega)$.

Proposition 3.2 will be proved if we can show that all the above terms in (3.1) and (3.2) belong to $L_{\text {loc }}^{2}(\Omega)$. Obviously, the terms in (3.1) are continuous and therefore they are in $L_{\text {loc }}^{2}(\Omega)$ since $a_{\alpha} \in C^{\infty}, \Phi \in C^{\infty}, X^{\vartheta} f \in C(\Omega)$ for all multi-orders $\vartheta$ such that $|\vartheta| \leqslant q$. For estimating the terms in (3.2) we put $r=\max \left\{\left|\iota^{1}\right|, \ldots,\left|\iota^{p}\right|\right\} \geq 1$. Choose one $j_{0}$ such that $r=\left|\iota^{j_{0}}\right|$. Consider the following possibilities:

A) $m_{j_{0}} \geq 2$. We state that $\left|\iota^{j}\right| \leqslant\left[\frac{m-q}{2}\right]$ for each $j=1, \ldots, p$, where [.] denotes the integral part of the argument. Indeed, if $j \neq j_{0}$ and $\left|\iota^{j}\right|>\left[\frac{m-q}{2}\right]$, then $\left|\iota^{j_{0}}\right| \geq$ $\left|\iota^{j}\right|>\left[\frac{m-q}{2}\right]$. Hence, $\left|\iota^{j}\right|+\left|\iota^{j_{0}}\right|>m-q$, and we come to a contradiction. If $j=j_{0}$ and $\left|\iota^{j_{0}}\right|>\left[\frac{m-q}{2}\right]$, then $\left|\iota^{j_{0}}\right| m_{j_{0}} \geq 2\left|\iota^{j_{0}}\right|>m-q$, which is impossible. Since $m \geq$ $n l+q+1$, all the factors $X^{\iota^{j}} X^{\vartheta^{j}} f$ in the terms (3.2) belong to $S_{\mathrm{loc}}^{\left[\frac{m-q}{2}\right],\left\{X_{j}\right\}_{j=1}^{k}}(\Omega) \subset$ $C(\Omega)$ for each $j=\overline{1, p}$. Thus, we conclude that the terms are in $C(\Omega)$, and therefore they belong to $L_{\text {loc }}^{2}(\Omega)$.

B) $m_{j_{0}}=1$ and there are no other elements $j$ such that $j \neq j_{0}$. Then the term will have form

$$
a_{\alpha, \beta}(x) \frac{\partial^{\alpha+\beta} \Phi\left(x, X^{\vartheta} f\right)_{|\vartheta| \leqslant q}}{\partial x^{\alpha} \partial \tau^{\beta}} X^{\iota^{j_{0}}} X^{\vartheta^{j_{0}}} f \in L_{\mathrm{loc}}^{2}(\Omega), \text { as }\left|\iota^{j_{0}}\right| \leqslant m-q .
$$

C) $m_{j_{0}}=1$ and there is at least one element $j_{1}$ such that $j_{1} \neq j_{0}$. As in part A) it is possible to establish that $\left|\iota^{j}\right| \leqslant\left[\frac{m-q}{2}\right]$ for all $j$, and therefore $X^{\iota^{j}} X^{\vartheta^{j}} f \in$ $S_{\text {loc }}^{\left[\frac{m-q}{2}\right],\left\{X_{j}\right\}_{j=1}^{k}}(\Omega) \subset C(\Omega)$ for all $j$. Thus,

$$
a_{\alpha, \beta}(x) \frac{\partial^{\alpha+\beta} \Phi\left(x, X^{\vartheta} f\right)_{|\vartheta| \leqslant q}}{\partial x^{\alpha} \partial \tau^{\beta}}\left(X^{\iota^{1}} X^{\vartheta^{1}} f\right)^{m_{1}} \cdots\left(X^{\iota^{p}} X^{\vartheta^{p}} f\right)^{m_{p}} \in C(\Omega) \subset L_{\mathrm{loc}}^{2}(\Omega) .
$$

The proof of Proposition 3.2 is complete.

Theorem 3.3. Assume that the vector fields $\left\{X_{j}\right\}_{j=1}^{k}$ satisfy the condition $(\mathrm{H})_{1}$ in $\Omega$ and $\Phi\left(x, f, \tau_{1}, \ldots, \tau_{k}\right)$ is an infinitely differentiable function. Then the nonlinear operator $\Psi_{2}\left(x, \partial^{\alpha}\right)$ is extendedly maximally hypoelliptic in the system $\left\{X_{j}\right\}_{j=1}^{k}$. In particular, it is hypoelliptic.

Proof. We show that if a function $f$ is a solution of the equation $\Psi_{2}\left(x, \partial^{\alpha}\right) f=g \in$ $S_{\mathrm{loc}}^{m_{1},\left\{X_{j}\right\}_{j=1}^{k}}(\Omega)$ and $f \in C^{n l+2}(\Omega)$, then $f \in S_{\mathrm{loc}}^{m_{1}+2,\left\{X_{j}\right\}_{j=1}^{k}}(\Omega)$ for each nonnegative integer $m_{1}$. In fact, assume that $f \in S_{\text {loc }}^{m,\left\{X_{j}\right\}_{j=1}^{k}}(\Omega)$ with $m \geq n l+2$. We have $\Phi\left(x, f, X_{1} f, \ldots, X_{k} f\right) \in S_{\mathrm{loc}}^{m-1,\left\{X_{j}\right\}_{j=1}^{k}}(\Omega)$ by Proposition 3.2 with $q=1$. Put 
$t=\min \left\{m-1, m_{1}\right\}$. Then from the equation

$$
\sum_{j=1}^{k} X_{j}^{2} f=g(x)-\Phi\left(x, f, X_{1} f, \ldots, X_{k} f\right) \in S_{\mathrm{loc}}^{t,\left\{X_{j}\right\}_{j=1}^{k}}(\Omega)
$$

and Theorem 3.1 it follows that $f \in S_{\mathrm{loc}}^{t+2,\left\{X_{j}\right\}_{j=1}^{k}}(\Omega)$. Repeating the above argument again and again, we obtain $f \in S_{\mathrm{loc}}^{m_{1}+2,\left\{X_{j}\right\}_{j=1}^{k}}(\Omega)$. Finally, note that the second statement in Theorem 3.3 is a straightforward consequence of the first statement and Remark 2.13. This concludes the proof of Theorem 3.3 .

Remark 3.4. Results of the same nature as in Theorem 3.3 were established in 20 . However, our method is different from the one in [20] which works in the Hölder settings.

Remark 3.5. In Theorem 3.3 the condition of semi-linearity of $\Psi_{2}$ is essential. Otherwise the theorem may not be true. For example, consider the following operator in $\mathbb{R}^{2}$ :

$$
\tilde{\Psi}=X_{1}^{2}+\left(X_{2}^{2}\right)^{2},
$$

where $X_{1}=\frac{\partial}{\partial x_{1}}, X_{2}=\frac{\partial}{\partial x_{2}}$. It is easy to see that

$$
\tilde{\Psi}\left(\left|x_{2}\right|^{2 m+1}\right)=(2 m+1)^{2}(2 m)^{2} x_{2}^{4 m-2} \in C^{\infty}\left(\mathbb{R}^{2}\right)
$$

for all $m \in \mathbb{Z}_{+}$. However $\left|x_{2}\right|^{2 m+1} \in C^{2 m}\left(\mathbb{R}^{2}\right) \backslash C^{\infty}\left(\mathbb{R}^{2}\right)$. Hence the nonlinear operator $\tilde{\Psi}$ is not hypoelliptic in $\mathbb{R}^{2}$.

\section{Semilinear hypoelliptic high order DifFerential EQUations}

\subsection{Maximal $\delta$-hypoellipticity and the $(\mathrm{K})_{1}$ condition.}

4.1.1. Linear operator. In this part we consider a linear operator of the form

$$
P_{2 m}:=P_{2 m}(x, \partial)=(-1)^{m} \sum_{j=1}^{k} X_{j}^{2 m}+\sum_{|\iota| \leqslant 2 m-1} a_{\iota}(x) X^{\iota},
$$

where $a_{\iota}(x) \in C^{\infty}(\Omega)$. In what follows, by small letters we will denote smooth functions, and by capital letters we will denote operators acting on a space of functions. Note that $P_{2}(x, \partial)$ is the Hörmander operator recalled in Section 2 . Here, the factor $(-1)^{m}$ is introduced for the reason of convenience in the computation process that arises in the future.

Lemma 4.1. Let $X \in T(\Omega)$. The following formula holds:

$$
\left(X^{\iota}\right)^{*}=(-1)^{|\iota|} X^{\bar{\iota}}+\sum_{\substack{\forall j: \vartheta(j) \leqslant \iota(j) \\|\vartheta|<|\iota|}} a_{\vartheta}(x) X^{\vartheta},
$$

where $\left(X^{\iota}\right)^{*}$ is the formal adjoint of $X^{\iota}$ and $\bar{\iota}=\left(\iota_{r}, \ldots, \iota_{1}\right)$ if $\iota=\left(\iota_{1}, \ldots, \iota_{r}\right)$.

Proof. This lemma can easily be proved by induction on $|\iota|$. We omit the details.

Now let $s \in \mathbb{R}$ and $\Lambda^{s}$ be the pseudodifferential operator (p.d.o.) with symbol $\left(1+|\xi|^{2}\right)^{\frac{s}{2}}$. If $K$ is a fixed compact in $\Omega$, then by $G^{s}:=G_{K}^{s}$ we denote the p.d.o. with the symbol $g(x)\left(1+|\xi|^{2}\right)^{\frac{s}{2}}$, where $g(x) \in C_{0}^{\infty}(\Omega), g(x) \equiv 1$ in some neighborhood 
of the compact $K$. It is well known that for each natural integer $N$ and any real number $s$ there exist a multi-functional $A_{N}$ and a constant $C=C(s, N)$ such that

$$
\begin{gathered}
(f, h)_{s}=\left(\Lambda^{s} f, \Lambda^{s} h\right)_{0}=\left(G^{s} f, G^{s} h\right)_{0}+A_{N}(f, h), \forall f, h \in C_{0}^{\infty}(K), \\
\left\|G^{s} f\right\|_{0}^{2}-C^{-1}\|f\|_{-N}^{2} \leqslant\|f\|_{s}^{2} \leqslant\left\|G^{s} f\right\|_{0}^{2}+C\|f\|_{-N}^{2}, \forall f \in C_{0}^{\infty}(K), \\
\left|A_{N}(f, h)\right| \leqslant C\left(\|f\|_{-N}^{2}+\|h\|_{-N}^{2}\right), \forall f, h \in C_{0}^{\infty}(K) .
\end{gathered}
$$

Lemma 4.2. Let $X \in T(\Omega)$. Then for any compact $K$ in $\Omega, \varepsilon>0, s \in \mathbb{R}, p \geq 1$ there exists a constant $C(\varepsilon)=C(\varepsilon, s, p, K)$ such that

$$
\left\|X^{p} f\right\|_{s}^{2} \leqslant \varepsilon\left\|X^{p+1} f\right\|_{s}^{2}+C(\varepsilon)\|f\|_{s}^{2}, \quad \forall f \in C_{0}^{\infty}(K) .
$$

Proof. For $p=1$ the lemma is trivial. Assume that it is true for $p \leqslant t$. We show it for $p=t+1$. Obviously, $X^{*}=-X+a(x)$. Therefore

$$
\begin{aligned}
\left\|X^{t+1} f\right\|_{s}^{2} & \leqslant\left(G^{s} X^{t+1} f, G^{s} X^{t+1} f\right)_{0}+C\|f\|_{s}^{2} \\
& =\left(X^{t} f,(-X+a(x)) G^{2 s} X^{t+1} f\right)_{0}+C\|f\|_{s}^{2} \\
& \leqslant \varepsilon_{1}\left\|X^{t+2} f\right\|_{s}^{2}+\frac{1}{4}\left\|X^{t+1} f\right\|_{s}^{2}+C\left(\varepsilon_{1}\right)\left\|X^{t} f\right\|_{s}^{2}+C\|f\|_{s}^{2} .
\end{aligned}
$$

Now choosing $\varepsilon_{1} \leqslant \frac{\varepsilon}{2}$ sufficiently small, by the inductive assumptions we conclude that

$$
\left\|X^{t+1} f\right\|_{s}^{2} \leqslant \frac{\varepsilon\left\|X^{t+2} f\right\|_{s}^{2}}{2}+\frac{\left\|X^{t+1} f\right\|_{s}^{2}}{2}+C(\varepsilon)\|f\|_{s}^{2},
$$

from which the lemma follows.

Lemma 4.3. Assume that the vector fields $\left\{X_{j}\right\}_{j=1}^{k}$ satisfy the condition $(\mathrm{H})_{1}$ in $\Omega$. Then for any compact $K$ in $\Omega$, any natural integer $i$ and each real number $s$ there exists a constant $C=C(l, s, K)$ such that

$$
\sum_{j=1}^{k}\left\|X_{j}^{i-1} f\right\|_{s}^{2} \leqslant C\left(\sum_{j=1}^{k}\left\|X_{j}^{i} f\right\|_{s-\frac{1}{l}}^{2}+\|f\|_{s-\frac{1}{l}}^{2}\right), \quad \forall f \in C_{0}^{\infty}(K) .
$$

Proof. For $i=1$ the estimate is true in view of Theorem 2.4. By assuming that the estimate (4.1) holds for $i \leqslant p$ we will prove it for $i=p+1$. We have

$$
\begin{aligned}
\sum_{j=1}^{k}\left\|X_{j}^{p} f\right\|_{0}^{2} & =\sum_{j=1}^{k}\left(X_{j}^{p} f, X_{j}^{p} f\right)_{0}=\sum_{j=1}^{k}\left(X_{j}^{p-1} f,\left(-X_{j}+a_{j}(x)\right) X_{j}^{p} f\right)_{0} \\
& \leqslant C(\varepsilon) \sum_{j=1}^{k}\left\|X_{j}^{p+1} f\right\|_{-\frac{1}{l}}^{2}+\varepsilon \sum_{j=1}^{k}\left\|X_{j}^{p-1} f\right\|_{\frac{1}{l}}^{2}+\frac{1}{2} \sum_{j=1}^{k}\left\|X_{j}^{p} f\right\|_{0}^{2}+C(\varepsilon)\|f\|_{-\frac{1}{l}}^{2},
\end{aligned}
$$

where $a_{j}(x) \in C^{\infty}(\Omega)$. From the just obtained estimate and the inductive assumptions, by choosing $\varepsilon$ sufficiently small, with the help of Lemma 4.2 we deduce (4.1) in the case $s=0$. It remains to prove the estimate in the general case. By Lemma 
2.14 and the inductive assumptions we have

$$
\begin{aligned}
\sum_{j=1}^{k}\left\|X_{j}^{p} f\right\|_{s}^{2} & \leqslant C\left(\sum_{j=1}^{k}\left\|X_{j}^{p} G^{s} f\right\|_{0}^{2}+\sum_{j=1}^{k}\|f\|_{s}^{2}\right) \\
& \leqslant C\left(\sum_{j=1}^{k}\left\|G^{s} X_{j}^{p+1} f\right\|_{-\frac{1}{l}}^{2}+\sum_{\substack{j=\overline{1, k} \\
i=1, p+1}}\left\|X_{j}^{p+1-i} f\right\|_{s-\frac{1}{l}}^{2}+\|f\|_{s}^{2}\right) \\
& \leqslant C\left(\sum_{j=1}^{k}\left\|X_{j}^{p+1} f\right\|_{s-\frac{1}{\tau}}^{2}+\|f\|_{s}^{2}\right)+\frac{1}{2} \sum_{j=1}^{k}\left\|X_{j}^{p} f\right\|_{s}^{2},
\end{aligned}
$$

which shows the correctness of (4.1) for arbitrary real $s \in \mathbb{R}$. Lemma 4.3 is now completely proved.

Corollary 4.4. Let the conditions of Lemma 4.3 be satisfied. Then the following inequalities hold:

$$
\begin{gathered}
\|f\|_{s+\frac{i}{l}}^{2} \leqslant C\left(\sum_{j=1}^{k}\left\|X_{j}^{i} f\right\|_{s}^{2}+\|f\|_{s}^{2}\right), \quad \forall f \in C_{0}^{\infty}(K), \\
\|f\|_{s+\frac{i}{l}}^{2} \leqslant C\left(\sum_{j=1}^{k}\left\|X_{j}^{i} f\right\|_{s}^{2}+\|f\|_{s^{\prime}}^{2}\right), \quad\left(s^{\prime}<s\right), \quad \forall f \in C_{0}^{\infty}(K), \\
\left\|X_{j_{1}}^{i_{1}} f\right\|_{s+\frac{i-i_{1}}{l}}^{2} \leqslant C\left(\sum_{j=1}^{k}\left\|X_{j}^{i} f\right\|_{s}^{2}+\|f\|_{s}^{2}\right), \quad i_{1} \leqslant i, j_{1}=\overline{1, k}, \forall f \in C_{0}^{\infty}(K), \\
\left\|X_{j_{1}} X_{j_{2}} f\right\|_{s+\frac{i-2}{l}}^{2} \leqslant C\left(\sum_{j=1}^{k}\left\|X_{j}^{i} f\right\|_{s}^{2}+\|f\|_{s}^{2}\right), \quad 2 \leqslant i, j_{1}, j_{2}=\overline{1, k}, \forall f \in C_{0}^{\infty}(K) .
\end{gathered}
$$

Lemma 4.5. Assume that the vector fields $\left\{X_{j}\right\}_{j=1}^{k}$ satisfy the conditions $(\mathrm{H})_{1}$ and $(\mathrm{K})_{1}^{\mathrm{d}}$ in $\Omega$. Then for any compact $K$ in $\Omega$, any arbitrary values $j_{1}, j_{2}=$ $\overline{1, k} ; i_{1}, i_{2}, i_{1}+i_{2} \leqslant d$, and each real number $s$ there exists a constant

$$
C\left(j_{1}, j_{2}, i_{1}, i_{2}, s, K\right)=C
$$

such that

$$
\left\|X_{j_{1}}^{i_{1}} X_{j_{2}}^{i_{2}} f\right\|_{s}^{2} \leqslant C\left(\sum_{j=1}^{k}\left\|X_{j}^{i_{1}+i_{2}} f\right\|_{s}^{2}+\|f\|_{s}^{2}\right), \forall f \in C_{0}^{\infty}(K) .
$$

Proof. For $i_{1}+i_{2}=1$ the lemma is trivial. For $i_{1}+i_{2}=2$ the lemma is true in view of Theorem 2.4] Assuming that the estimate (4.2) holds for $i_{1}+i_{2} \leqslant t \leqslant d-1$, we prove it for $i_{1}+i_{2}=t+1$. Clearly, we can suppose that $i_{1} \geq 1, i_{2} \geq 1$. By the inductive assumptions the following inequality holds:

$$
\begin{aligned}
\left\|X_{j_{1}}^{i_{1}} X_{j_{2}}^{i_{2}} f\right\|_{s}^{2} & =\left\|X_{j_{1}}^{i_{1}} X_{j_{2}}^{i_{2}-1}\left(X_{j_{2}} f\right)\right\|_{s}^{2} \leqslant C\left(\sum_{j=1}^{k}\left\|X_{j}^{i_{1}+i_{2}-1} X_{j_{2}} f\right\|_{s}^{2}+\left\|X_{j_{2}} f\right\|_{s}^{2}\right) \\
& \leqslant C\left(\sum_{j=1}^{k}\left\|X_{j}^{i_{1}+i_{2}} f\right\|_{s}^{2}+\sum_{j=1}^{k}\left\|X_{j}^{i_{1}+i_{2}-1} X_{j_{2}} f\right\|_{s}^{2}+\|f\|_{s}^{2}\right) .
\end{aligned}
$$


Therefore the matter reduces to proving the estimate (4.2) for terms of the forms $X_{j_{1}}^{t} X_{j_{2}} f$. By Lemma 2.14 we have

$$
\begin{gathered}
\left(X_{j_{1}}^{t} X_{j_{2}} f, X_{j_{1}}^{t} X_{j_{2}} f\right)_{0}=-\left(X_{j_{1}}^{t-1} X_{j_{2}} f, X_{j_{1}}^{t+1} X_{j_{2}} f\right)_{0}+\left(X_{j_{1}}^{t-1} X_{j_{2}} f, a_{j_{1}} X_{j_{1}}^{t} X_{j_{2}} f\right)_{0} \\
=-\left(X_{j_{1}}^{t-1} X_{j_{2}} f, X_{j_{2}} X_{j_{1}}^{t+1} f\right)_{0}-\sum_{i=1}^{t+1} C_{t+1}^{i}\left(X_{j_{1}}^{t-1} X_{j_{2}} f,\left[{ }_{i} X_{j_{1}}, X_{j_{2}}\right] X_{j_{1}}^{t+1-i} f\right)_{0}+I_{1} \\
:=J_{0}+\sum_{i=1}^{t+1} J_{i}+I_{1} .
\end{gathered}
$$

Using the inductive assumptions and the Cauchy-Schwarz inequality we obtain

$$
\left|I_{1}\right| \leqslant \varepsilon\left\|X_{j_{1}}^{t} X_{j_{2}} f\right\|_{0}^{2}+C(\varepsilon)\left(\sum_{j=1}^{k}\left\|X_{j}^{t} f\right\|_{0}^{2}+\|f\|_{0}^{2}\right) .
$$

In view of the condition $(\mathrm{K})_{1}^{\mathrm{d}}$ of the lemma, taking into account the inductive assumptions and the estimate (4.3) we deduce that

$$
\begin{aligned}
\left|J_{1}\right| & \leqslant C(\varepsilon)\left\|X_{j_{1}}^{t-1} X_{j_{2}} f\right\|_{\frac{1}{\tau}}^{2}+\varepsilon\left(\sum_{j=1}^{k}\left\|X_{j} X_{j_{1}}^{t} f\right\|_{0}^{2}+\left\|X_{j_{1}}^{t} f\right\|_{0}^{2}\right) \\
& \leqslant C(\varepsilon)\left(\sum_{j=1}^{k}\left\|X_{j}^{t+1} f\right\|_{0}^{2}+\|f\|_{0}^{2}\right)+\varepsilon \sum_{j=1}^{k}\left\|X_{j}^{t} X_{j_{1}} f\right\|_{0}^{2} .
\end{aligned}
$$

Further, for $2 \leqslant i \leqslant t+1$, by using the condition $(\mathrm{K})_{1}^{\mathrm{d}}$ it can be checked that

$$
\begin{aligned}
\left|J_{i}\right| & \leqslant C\left(\left\|X_{j_{1}}^{t-1} X_{j_{2}} f\right\|_{0}^{2}+\left\|\left[{ }_{i} X_{j_{1}}, X_{j_{2}}\right] X_{j_{1}}^{t+1-i} f\right\|_{0}^{2}\right) \\
& \leqslant C\left(\sum_{j=1}^{k}\left\|X_{j}^{t} f\right\|_{0}^{2}+\sum_{j=1}^{k}\left\|X_{j} X_{j_{1}}^{t+1-i} f\right\|_{\frac{i}{l}}^{2}+\|f\|_{0}^{2}\right) \\
& \leqslant C\left(\sum_{j=1}^{k}\left\|X_{j}^{t+2-i} f\right\|_{0}^{2}+\|f\|_{0}^{2}\right) \leqslant C\left(\sum_{j=1}^{k}\left\|X_{j}^{t} f\right\|_{0}^{2}+\|f\|_{0}^{2}\right) .
\end{aligned}
$$

Finally, we estimate $J_{0}$. We have

$$
\begin{aligned}
J_{0} & =\left(X_{j_{2}} X_{j_{1}}^{t-1} X_{j_{2}} f, X_{j_{1}}^{t+1} f\right)_{0}-\left(a_{j_{2}} X_{j_{1}}^{t-1} X_{j_{2}} f, X_{j_{1}}^{t+1} f\right)_{0} \\
& \leqslant C(\varepsilon)\left\|X_{j_{1}}^{t+1} f\right\|_{0}^{2}+\varepsilon\left\|X_{j_{2}} X_{j_{1}}^{t-1} X_{j_{2}} f\right\|_{0}^{2}+C\left\|X_{j_{1}}^{t-1} X_{j_{2}} f\right\|_{0}^{2} \\
& \leqslant C(\varepsilon)\left(\sum_{j=1}^{k}\left\|X_{j}^{t+1} f\right\|_{0}^{2}+\|f\|_{0}^{2}\right)+\varepsilon \sum_{j=1}^{k}\left\|X_{j}^{t} X_{j_{2}} f\right\|_{0}^{2} .
\end{aligned}
$$

Altogether, from the obtained inequalities we find that

$$
\left\|X_{j_{1}}^{t} X_{j_{2}} f\right\|_{0}^{2} \leqslant C(\varepsilon)\left(\sum_{j=1}^{k}\left\|X_{j}^{t+1} f\right\|_{0}^{2}+\|f\|_{0}^{2}\right)+\varepsilon \sum_{j, j^{\prime}=1}^{k}\left\|X_{j}^{t} X_{j^{\prime}} f\right\|_{0}^{2} .
$$

By setting $M=\max _{j, j^{\prime}=1, k}\left\|X_{j}^{t} X_{j^{\prime}} f\right\|_{0}^{2}$, from the last inequality we have

$$
\left\|X_{j_{1}}^{t} X_{j_{2}} f\right\|_{0}^{2} \leqslant M \leqslant C(\varepsilon)\left(\sum_{j=1}^{k}\left\|X_{j}^{t+1} f\right\|_{0}^{2}+\|f\|_{0}^{2}\right)+k^{2} \varepsilon M .
$$


If we take $\varepsilon=\frac{k^{2}}{2}$, then

$$
\left\|X_{j_{1}}^{t} X_{j_{2}} f\right\|_{0}^{2} \leqslant C\left(\sum_{j=1}^{k}\left\|X_{j}^{t+1} f\right\|_{0}^{2}+\|f\|_{0}^{2}\right) .
$$

This is the estimate (4.2) in the case $s=0$. We begin to prove the lemma in the general case by substituting $G^{s} f$ for $f$ in (4.4):

$$
\begin{gathered}
\left\|X_{j_{1}}^{t} X_{j_{2}} G^{s} f\right\|_{0}^{2} \leqslant C\left(\sum_{j=1}^{k}\left\|X_{j}^{t+1} G^{s} f\right\|_{0}^{2}+\left\|G^{s} f\right\|_{0}^{2}\right) \\
\leqslant C\left(\sum_{j=1}^{k}\left\|G^{s} X_{j}^{t+1} f\right\|_{0}^{2}+\sum_{i=1}^{t+1} \sum_{j=1}^{k}\left\|X_{j}^{t+1-i} f\right\|_{s}^{2}\right) \leqslant C\left(\sum_{j=1}^{k}\left\|X_{j}^{t+1} f\right\|_{s}^{2}+\|f\|_{s}^{2}\right) .
\end{gathered}
$$

On the other hand, by Lemma 2.16

$$
\begin{aligned}
\left\|X_{j_{1}}^{t} X_{j_{2}} f\right\|_{s}^{2} & \leqslant C\left(\left\|G^{s} X_{j_{1}}^{t} X_{j_{2}} f\right\|_{0}^{2}+\|f\|_{s}^{2}\right) \leqslant C\left(\left\|X_{j_{1}}^{t} X_{j_{2}} G^{s} f\right\|_{0}^{2}\right. \\
& \left.+\sum_{(0,0)<\left(i_{3}, i_{4}\right) \leqslant(t, 1)} C_{(t, 1)}^{\left(i_{3}, i_{4}\right)}\left\|\left[i_{3} X_{j_{1}}\left[i_{4} X_{j_{2}}, G^{s}\right]\right] X_{j_{1}}^{t-i_{3}} X_{j_{2}}^{1-i_{4}} f\right\|_{0}^{2}\right) \\
& \leqslant C\left(\left\|X_{j_{1}}^{t} X_{j_{2}} G^{s} f\right\|_{0}^{2}+\sum_{j=1}^{k}\left\|X_{j}^{t} f\right\|_{s}^{2}+\|f\|_{s}^{2}\right) .
\end{aligned}
$$

From the last two inequalities we immediately get the desired result.

Remark 4.6. Note that Lemma 4.3 remains true if we replace the condition $(\mathrm{K})_{1}^{\mathrm{d}}$ by the condition $\left(\mathrm{K}^{\prime}\right)_{1}^{\mathrm{d}}$.

Lemma 4.7. Assume that the vector fields $\left\{X_{j}\right\}_{j=1}^{k}$ satisfy the conditions $(\mathrm{H})_{1}$ and $(\mathrm{K})_{1}^{\mathrm{d}}\left(\right.$ or $\left.\left(\mathrm{K}^{\prime}\right)_{1}^{\mathrm{d}}\right)$ in $\Omega$. Then for any compact $K$ in $\Omega$, any values $j_{1}, j_{2}=$ $\overline{1, k} ; q, i_{1}, \ldots, i_{2 q-1}$ with $i_{1}+\cdots i_{2 q-1} \leqslant d$, and each real number $s$, there exists a constant $C\left(j_{1}, j_{2}, q, i_{1}, \ldots, i_{2 q-1}, s, K\right)=C$ such that

$$
\left\|X_{j_{1}}^{i_{1}} X_{j_{2}}^{i_{2}} \ldots X_{j_{1}}^{i_{2 q-1}} f\right\|_{s}^{2} \leqslant C\left(\sum_{j=1}^{k}\left\|X_{j}^{i_{1}+\cdots+i_{2 q-1}} f\right\|_{s}^{2}+\|f\|_{s}^{2}\right)
$$

for all $f \in C_{0}^{\infty}(K)$.

Proof. For $q=1$ the estimate (4.5) is trivial. Suppose that it is true for $q \leqslant t$. Then we show that it is true for terms of the forms

$$
X_{j_{1}}^{i_{1}} X_{j_{2}}^{i_{2}} \ldots X_{j_{2}}^{i_{2 t}} f\left(i_{2 t} \geq 1\right), X_{j_{1}}^{i_{1}} X_{j_{2}}^{i_{2}} \ldots X_{j_{2}}^{i_{2 q}} X_{j_{1}}^{i_{2 t+1}} f\left(i_{2 t+1} \geq 1\right) .
$$

Indeed, by the inductive assumption, from Lemma 4.5 we deduce that

$$
\begin{gathered}
\left\|X_{j_{1}}^{i_{1}} X_{j_{2}}^{i_{2}} \ldots X_{j_{2}}^{i_{2 t}} f\right\|_{s}^{2}=\left\|X_{j_{1}}^{i_{1}} X_{j_{2}}^{i_{2}} \ldots X_{j_{1}}^{i_{2 t-1}}\left(X_{j_{2}}^{i_{2 t}} f\right)\right\|_{s}^{2} \\
\leqslant C\left(\sum_{j=1}^{k}\left\|X_{j}^{i_{1}+\cdots+i_{2 t-1}}\left(X_{j_{2}}^{i_{2 t}} f\right)\right\|_{s}^{2}+\left\|X_{j_{2}}^{i_{2 t}} f\right\|_{s}^{2}\right) \leqslant C\left(\sum_{j=1}^{k}\left\|X_{j}^{i_{1}+\cdots+i_{2 t}} f\right\|_{s}^{2}+\|f\|_{s}^{2}\right) .
\end{gathered}
$$

Having established the estimate for $X_{j_{1}}^{i_{1}} X_{j_{2}}^{i_{2}} \ldots X_{j_{2}}^{i_{2 t}} f$, we can then get the estimate for $X_{j_{1}}^{i_{1}} X_{j_{2}}^{i_{2}} \ldots X_{j_{2}}^{i_{2 q}} X_{j_{1}}^{i_{2 t+1}} f$ in a similar way. Thus, Lemma 4.7 is proved. 
Remark 4.8. The estimate (4.5) is true for $X_{j_{1}}^{i_{1}} X_{j_{2}}^{i_{2}} \ldots X_{j_{2}}^{i_{2 q}} f$ since we can write $X_{j_{1}}^{i_{1}} X_{j_{2}}^{i_{2}} \ldots X_{j_{2}}^{i_{2 q}} f=X_{j_{1}}^{i_{1}} X_{j_{2}}^{i_{2}} \ldots X_{j_{2}}^{i_{2 q}} X_{j_{1}}^{i_{2 t+1}} f$ with $i_{2 t+1}=0$. Alternatively, it is also clear from the proof of Lemma 4.7

Lemma 4.9. Assume that the vector fields $\left\{X_{j}\right\}_{j=1}^{k}$ satisfy the conditions $(\mathrm{H})_{1}$ and $(\mathrm{K})_{1}^{m}$ in $\Omega$. For any compact $K$ in $\Omega$ and any real number $s$ there exists a constant $C=C(s, K)$ such that

$$
\sum_{j=1}^{k}\left\|X_{j}^{m} f\right\|_{s}^{2} \leqslant 2 \operatorname{Re}\left(P_{2 m} f, f\right)_{s}+C\|f\|_{s}^{2}
$$

for all $f \in C_{0}^{\infty}(K)$.

Proof. We begin by rewriting $a_{\iota} X^{\iota}$ in $P_{2 m}(x, \partial)$ as $a_{\iota} X^{\iota^{1}} X^{\iota^{2}}$ where $\left|\iota^{1}\right| \leqslant m-$ $1,\left|\iota^{2}\right| \leqslant m$. Therefore using Lemmas 2.17 and 4.7 we obtain

$$
\begin{aligned}
\left(P_{2 m} f, f\right)_{s} & =A_{N}\left(P_{2 m} f, f\right)+\left(G^{s} P_{2 m} f, G^{s} f\right)_{0}=A_{N}\left(P_{2 m} f, f\right) \\
& +(-1)^{m} \sum_{j=1}^{k}\left(X_{j}^{m} G^{s} X_{j}^{m} f, G^{s} f\right)_{0} \\
& +\sum_{j=1}^{k} \sum_{i=1}^{m} C_{m}^{i}(-1)^{m}\left(\left[G^{s},{ }_{i} X_{j}\right] X_{j}^{m} f,\left(X_{j}^{m-i}\right)^{*} G^{s} f\right)_{0} \\
& +\sum_{|\iota| \leqslant 2 m-1} \sum_{\iota^{3} \subseteq \iota^{1}, \iota^{3} \neq \iota^{1}}\left(\left[G^{s}, \iota_{\iota} \backslash \iota^{3} X\right] X^{\iota^{2}} f,\left(X^{\iota^{3}}\right)^{*} a_{\iota}^{*}(x) G^{s} f\right)_{0} \\
& +\sum_{|\iota| \leqslant 2 m-1} \sum_{\iota^{3} \subseteq \iota^{1}, \iota^{3} \neq \iota^{1}}\left(\left[\left[G^{s}, a_{\iota}\right], \iota_{\iota^{1} \backslash \iota^{3}} X\right] X^{\iota^{2}} f,\left(X^{\iota^{3}}\right)^{*} G^{s} f\right)_{0} \\
& :=A_{N}\left(P_{2 m} f, f\right)+I_{1}+I_{2}+I_{3}+I_{4} .
\end{aligned}
$$

First we estimate $I_{1}$. We have

$$
\begin{aligned}
I_{1} & =(-1)^{m} \sum_{j=1}^{k}\left(G^{s} X_{j}^{m} f, X_{j}^{* m} G^{s} f\right)_{0}=\sum_{j=1}^{m}\left\|G^{s} X_{j}^{m} f\right\|_{0}^{2} \\
& +(-1)^{m} \sum_{j=1}^{k} \sum_{i=0}^{m-1}\left(G^{s} X_{j}^{m} f, G^{s} a_{i, j} X_{j}^{i} f\right)_{0} \\
& +(-1)^{m} \sum_{j=1}^{k} \sum_{i=1}^{m} C_{m}^{i}\left(G^{s} X_{j}^{m} f,\left[{ }_{i} X_{j}^{*}, G^{s}\right]\left(X_{j}^{*}\right)^{m-i} f\right)_{0} \\
& :=\sum_{j=1}^{m}\left\|G^{s} X_{j}^{m} f\right\|_{0}^{2}+J_{1}+J_{2} .
\end{aligned}
$$

Since the order of the operator $\left[{ }_{i} X_{j}^{*}, G^{s}\right]$ equals $s$, for any $\varepsilon>0$ the following estimate holds:

$$
\max \left\{\left|J_{1}\right|,\left|J_{2}\right|\right\} \leqslant \varepsilon \sum_{j=1}^{m}\left\|G^{s} X_{j}^{m} f\right\|_{0}^{2}+C(\varepsilon)\|f\|_{s}^{2} .
$$


Analogously, it can be shown that

$$
\max \left\{\left|I_{2}\right|,\left|I_{3}\right|,\left|I_{4}\right|\right\} \leqslant \varepsilon \sum_{j=1}^{m}\left\|G^{s} X_{j}^{m} f\right\|_{0}^{2}+C(\varepsilon)\|f\|_{s}^{2} .
$$

Thus,

$$
\begin{aligned}
\sum_{j=1}^{m}\left\|G^{s} X_{j}^{m} f\right\|_{0}^{2} & =\operatorname{Re}\left(P_{2 m} f, f\right)_{s}-\operatorname{Re}\left(A_{N}\left(P_{2 m} f, f\right)+J_{1}+J_{2}+I_{2}+I_{3}+I_{4}\right) \\
& \leqslant \operatorname{Re}\left(P_{2 m} f, f\right)_{s}+\left|A_{N}\left(P_{2 m} f, f\right)\right|+\left|J_{1}\right|+\left|J_{2}\right|+\left|I_{2}\right|+\left|I_{3}\right|+\left|I_{4}\right| \\
& \leqslant \operatorname{Re}\left(P_{2 m} f, f\right)_{s}+\varepsilon \sum_{j=1}^{m}\left\|G^{s} X_{j}^{m} f\right\|_{0}^{2}+C(\varepsilon)\|f\|_{s}^{2} .
\end{aligned}
$$

Choosing $\varepsilon$ sufficiently small we get the desired result.

Theorem 4.10. Assume that the vector fields $\left\{X_{j}\right\}_{j=1}^{k}$ satisfy the conditions $(\mathrm{H})_{1}$ and $(\mathrm{K})_{1}^{3 m}$ (or $\left(\mathrm{K}^{\prime}\right)_{1}^{3 m}$ ) in $\Omega$. Then for any compact $K$ in $\Omega$, any real value $s$ there exists a constant $C(s, K)=C$ such that

$$
\sum_{j=1}^{k}\left\|X_{j}^{i} f\right\|_{s+\frac{2 m-i}{l}}^{2} \leqslant C\left(\left\|P_{2 m} f\right\|_{s}^{2}+\|f\|_{s}^{2}\right), \quad \forall f \in C_{0}^{\infty}(K),
$$

where $i=0,1, \ldots, 2 m$.

Proof. By Lemma 4.9 and Corollary 4.4, we have

$$
\begin{aligned}
\sum_{j=1}^{k}\left\|X_{j}^{m} f\right\|_{s+\frac{m}{l}}^{2} & \leqslant C\left(\operatorname{Re}\left(P_{2 m} f, f\right)_{s+\frac{m}{l}}+\|f\|_{s+\frac{m}{l}}^{2}\right) \\
& \leqslant C\left(\left\|P_{2 m} f\right\|_{s}+\|f\|_{s+\frac{m}{l}}\right)\|f\|_{s+\frac{2 m}{l}} \\
& \leqslant C\left(\left\|P_{2 m} f\right\|_{s}+\|f\|_{s+\frac{m}{l}}\right)\left(\sum_{j=1}^{k}\left\|X_{j}^{m} f\right\|_{s+\frac{m}{l}}^{2}+\|f\|_{s+\frac{m}{l}}^{2}\right)^{\frac{1}{2}}
\end{aligned}
$$

so that

$$
\sum_{j=1}^{k}\left\|X_{j}^{m} f\right\|_{s+\frac{m}{l}}^{2} \leqslant C\left(\left\|P_{2 m} f\right\|_{s}^{2}+\|f\|_{s+\frac{m}{l}}^{2}\right) .
$$

Now, if in the estimate

$$
\|f\|_{s+\frac{m}{l}}^{2} \leqslant \varepsilon\|f\|_{s+\frac{2 m}{l}}^{2}+C(\varepsilon)\|f\|_{s}^{2} \leqslant \varepsilon \sum_{j=1}^{k}\left\|X_{j}^{m} f\right\|_{s+\frac{m}{l}}^{2}+C(\varepsilon)\|f\|_{s}^{2}
$$

we choose $\varepsilon$ sufficient small, then from (4.7) we obtain

$$
\sum_{j=1}^{k}\left\|X_{j}^{m} f\right\|_{s+\frac{m}{l}}^{2} \leqslant \frac{1}{2} \sum_{j=1}^{k}\left\|X_{j}^{m} f\right\|_{s+\frac{m}{l}}^{2}+C\left(\left\|P_{2 m} f\right\|_{s}^{2}+\|f\|_{s}^{2}\right) .
$$

That is,

$$
\sum_{j=1}^{k}\left\|X_{j}^{m} f\right\|_{s+\frac{m}{l}}^{2} \leqslant C\left(\left\|P_{2 m} f\right\|_{s}^{2}+\|f\|_{s}^{2}\right)
$$


By using Lemma 4.3, we finally conclude that

$$
\begin{aligned}
\sum_{j=1}^{k}\left\|X_{j}^{i} f\right\|_{s+\frac{2 m-i}{l}}^{2} & \leqslant C\left(\sum_{j=1}^{k}\left\|X_{j}^{m} f\right\|_{s+\frac{m}{l}}^{2}+\|f\|_{s}^{2}\right) \\
& \leqslant C\left(\left\|P_{2 m} u\right\|_{s}^{2}+\|f\|_{s}^{2}\right), \quad i=0,1, \ldots, m
\end{aligned}
$$

Thus, the estimate (4.6) is proved for $i=0,1, \ldots, m$. We will show the estimate (4.6) for $i=m+1, \ldots, 2 m$. We will prove it by induction on $m+i$, for $i=0, \ldots, m$. For $i=0$ the estimate (4.6) is true in view of (4.8). Assuming that it is true for $i=i_{0} \leqslant m-1$ we show its availability for $i=i_{0}+1$. In fact, taking the scalar product $(-1)^{i_{0}+1}\left(P_{2 m} f, X_{1}^{2\left(i_{0}+1\right)} f\right)_{0}$, integrating by parts and with the help of Lemma 4.1 one obtain the following equality:

$$
\begin{aligned}
\left\|X_{1}^{m+i_{0}+1} f\right\|_{0}^{2} & =(-1)^{i_{0}+1}\left(P_{2 m} f, X_{1}^{2\left(i_{0}+1\right)} f\right)_{0}+(-1)^{m+i_{0}} \sum_{j=2}^{k}\left(X_{j}^{2 m} f, X_{1}^{2 i_{0}+2} f\right)_{0} \\
& +(-1)^{i_{0}} \sum_{|\iota| \leqslant 2 m-1}\left(a_{\iota} X^{\iota} f, X_{1}^{2 i_{0}+2} f\right)_{0} \\
& +\sum_{q=1}^{m-i_{0}-1}\left(X_{1}^{m+i_{0}+1} f, b_{q} X_{1}^{m+i_{0}+1-q} f\right)_{0}:=I_{1}+I_{2}+I_{3}+I_{4} .
\end{aligned}
$$

First we calculate $I_{2}$. From Lemma 4.1, it follows that

$$
I_{2}:=(-1)^{i_{0}} \sum_{j=2}^{k}\left(X_{j}^{m} f, X_{j}^{m} X_{1}^{2 i_{0}+2} f\right)_{0}+I_{21} .
$$

Consider the terms of the highest order in (4.9). We have

$$
\begin{gathered}
(-1)^{i_{0}}\left(X_{j}^{m} f, X_{j}^{m} X_{1}^{2 i_{0}+2} f\right)_{0}=-\left\|X_{1}^{i_{0}+1} X_{j}^{m} f\right\|_{0}^{2}+\sum_{q=1}^{i_{0}+1}\left(d_{q} X_{1}^{i_{0}+1-q} X_{j}^{m} f, X_{1}^{i_{0}+1} X_{j}^{m} f\right)_{0} \\
+(-1)^{i_{0}}\left(X_{j}^{m} f,\left[X_{j}^{m}, X_{1}^{2 i_{0}+2}\right] f\right)_{0}:=-\left\|X_{1}^{i_{0}+1} X_{j}^{m} f\right\|_{0}^{2}+I_{22}+I_{23} .
\end{gathered}
$$

Further, for any $\varepsilon>0$ there exists a constant $C(\varepsilon)$ such that

$$
\begin{aligned}
\left|I_{22}\right| & \leqslant \varepsilon\left\|X_{1}^{i_{0}+1} X_{j}^{m} f\right\|_{0}^{2}+C(\varepsilon) \sum_{q=1}^{i_{0}+1}\left\|X_{1}^{i_{0}+1-q} X_{j}^{m} f\right\|_{0}^{2} \\
& \leqslant \varepsilon \sum_{j=1}^{k}\left\|X_{j}^{i_{0}+1+m} f\right\|_{0}^{2}+C(\varepsilon)\left(\sum_{j=1}^{k}\left\|X_{j}^{i_{0}+m} f\right\|_{0}^{2}+\|f\|_{0}^{2}\right) .
\end{aligned}
$$


Taking into account Lemmas 2.18 and 4.1, we conclude that

$$
\begin{aligned}
\left|I_{23}\right| & =\left|\sum_{\substack{\left|\iota^{1}\right|+\left|\iota^{2}\right| \leqslant m+2 i_{0}+2 \\
\left|\iota^{2}\right| \geq 2}} C\left(\iota^{1}, \iota^{2}\right)\left(X_{j}^{m} f, X_{\iota^{2}} X^{\iota^{1}} f\right)_{0}\right| \\
& \leqslant C \sum_{\substack{\left|\iota^{2}\right|+\left|\iota^{3}\right|+\left|\iota^{4}\right| \leqslant m+2 i_{0}+2,\left|\iota^{2}\right| \geq 2,\left|\iota^{3}\right| \leqslant m+i_{0}+1,\left|\iota^{4}\right| \leqslant i_{0}-1}}\left|\left(e_{\iota^{4}} X^{\iota^{4}} X_{\iota^{2}}^{*} X_{j}^{m} f, X^{\iota^{3}} f\right)_{0}\right| \\
& \leqslant C \sum_{\substack{\left|\iota^{6}\right| \leqslant i_{0}-1,\left|\iota^{3}\right|+\left|\iota^{5}\right|+\left|\iota^{6}\right| \leqslant m+2 i_{0}+2,\left|\iota^{5}\right| \leqslant 3 m,\left|\iota^{3}\right| \leqslant m+i_{0}+1}}\left|\left(v_{\iota^{5}, \iota^{6}} X_{\iota^{5}} X^{\iota^{6}} X_{j}^{m} f, X^{\iota^{3}} f\right)_{0}\right| \\
& \leqslant \varepsilon \sum_{j=1}^{k}\left\|X_{j}^{m+i_{0}+1} f\right\|_{0}^{2}+C(\varepsilon)\left(\sum_{j=1}^{k}\left\|X_{j}^{m+i_{0}} f\right\|_{\frac{1}{l}}^{2}+\|f\|_{0}^{2}\right),
\end{aligned}
$$

in which $\varepsilon$ is any positive number. In the same way we can get

$$
\max \left\{\left|I_{21}\right|,\left|I_{3}\right|,\left|I_{4}\right|\right\} \leqslant \varepsilon \sum_{j=1}^{k}\left\|X_{j}^{m+i_{0}+1} f\right\|_{0}^{2}+C(\varepsilon)\left(\sum_{j=1}^{k}\left\|X_{j}^{m+i_{0}} f\right\|_{\frac{1}{l}}^{2}+\|f\|_{0}^{2}\right),
$$

for any positive $\varepsilon$. Thus, it is shown that

$$
\begin{aligned}
\left\|X_{1}^{m+i_{0}+1} f\right\|_{0}^{2} & =(-1)^{i_{0}+1} \operatorname{Re}\left(P_{2 m} f, X_{1}^{2\left(i_{0}+1\right)} f\right)_{0}-\left\|X_{1}^{i_{0}+1} X_{j}^{m} f\right\|_{0}^{2}+\operatorname{Re} I_{21} \\
& +\operatorname{Re} I_{22}+\operatorname{Re} I_{23}+\operatorname{Re} I_{3}+\operatorname{Re} I_{4} \leqslant(-1)^{i_{0}+1} \operatorname{Re}\left(P_{2 m} f, X_{1}^{2\left(i_{0}+1\right)} f\right)_{0} \\
& +\left|I_{21}\right|+\left|I_{22}\right|+\left|I_{23}\right|+\left|I_{3}\right|+\left|I_{4}\right| \leqslant(-1)^{i_{0}+1} \operatorname{Re}\left(P_{2 m} f, X_{1}^{2\left(i_{0}+1\right)} f\right)_{0} \\
& +\varepsilon \sum_{j=1}^{k}\left\|X_{j}^{m+i_{0}+1} f\right\|_{0}^{2}+C(\varepsilon)\left(\sum_{j=1}^{k}\left\|X_{j}^{m+i_{0}} f\right\|_{\frac{1}{l}}^{2}+\|f\|_{0}^{2}\right) .
\end{aligned}
$$

Similarly, the following inequalities hold:

$$
\begin{aligned}
\left\|X_{j}^{m+i_{0}+1} f\right\|_{0}^{2} & \leqslant(-1)^{i_{0}+1} \operatorname{Re}\left(P_{2 m} f, X_{j}^{2 i_{0}+2} f\right)_{0} \\
& +\varepsilon \sum_{j=1}^{k}\left\|X_{j}^{m+i_{0}+1} f\right\|_{0}^{2}+C(\varepsilon)\left(\sum_{j=1}^{k}\left\|X_{j}^{m+i_{0}} f\right\|_{\frac{1}{\tau}}^{2}+\|f\|_{0}^{2}\right), j=2, \ldots, k .
\end{aligned}
$$

Summing up in $j$ from 1 to $k$, one obtains

$$
\begin{aligned}
\sum_{j=1}^{k}\left\|X_{j}^{m+i_{0}+1} f\right\|_{0}^{2} & \leqslant(-1)^{i_{0}+1} \operatorname{Re}\left(P_{2 m} f, \sum_{j=1}^{k} X_{j}^{2 i_{0}+2} f\right)_{0} \\
& +k \varepsilon \sum_{j=1}^{k}\left\|X_{j}^{m+i_{0}+1} f\right\|_{0}^{2}+C(\varepsilon)\left(\sum_{j=1}^{k}\left\|X_{j}^{m+i_{0}} f\right\|_{\frac{1}{\tau}}^{2}+\|f\|_{0}^{2}\right) .
\end{aligned}
$$

Choosing $\varepsilon=\frac{1}{2 k}$, we immediately deduce that

$$
\sum_{j=1}^{k}\left\|X_{j}^{m+i_{0}+1} f\right\|_{0}^{2} \leqslant C\left(\left\|P_{2 m} f\right\|_{-\frac{m-\left(i_{0}+1\right)}{l}}^{2}+\|f\|_{0}^{2}\right) .
$$


Replacing $f$ by $G^{s+\frac{m-i_{0}-1}{l}} f$ in the just obtained estimate, in view of the result in the first part and by the inductive assumptions we get

$$
\sum_{j=1}^{k}\left\|X_{j}^{m+i_{0}+1} f\right\|_{s+\frac{m-i_{0}-1}{l}}^{2} \leqslant C\left(\left\|P_{2 m} f\right\|_{s}^{2}+\|f\|_{s}^{2}\right),
$$

which is exactly what we need to obtain.

Remark 4.11. In the course of the proof of Theorem4.10 it is clear that the stronger estimates

$$
\begin{gathered}
\sum_{j=1}^{k}\left\|X_{j}^{m+p} f\right\|_{s}^{2} \leqslant 2 \sum_{i=0}^{p} \operatorname{Re}\left(P_{2 m} f,(-1)^{i} \sum_{j=1}^{k} X_{j}^{2 i} f\right)_{s+\frac{p-i}{l}}+C\|f\|_{s}^{2}, \\
p=0,1, \ldots, m
\end{gathered}
$$

are still true.

Theorem 4.12. Assume that the vector fields $\left\{X_{j}\right\}_{j=1}^{k}$ satisfy the conditions $(\mathrm{H})_{1}$ and $(\mathrm{K})_{1}^{3 m}$ (or $\left(\mathrm{K}^{\prime}\right)_{1}^{3 m}$ ) in $\Omega$. Then for any compact $K$ in $\Omega$, and any real number $s$ there exists a constant $C(s, K)=C$ such that

$$
\sum_{|\iota| \leqslant 2 m}\left\|X^{\iota} f\right\|_{s+\frac{2 m-|\iota|}{l}}^{2} \leqslant C\left(\left\|P_{2 m} f\right\|_{s}^{2}+\|f\|_{s}^{2}\right), \quad \forall f \in C_{0}^{\infty}(K) .
$$

In other words, $P_{2 m}$ is maximally $\frac{1}{l}$-hypoelliptic.

Proof. Using Corollary 4.4, Lemma 4.7 and Theorem 4.10 we immediately get the desired result.

Comparing Remark 2.7 to Theorem 4.12 one obtains

Theorem 4.13. Assume that the vector fields $\left\{X_{j}\right\}_{j=1}^{k}$ satisfy the conditions $(\mathrm{H})_{1}$ and $(\mathrm{K})_{1}^{3 m}$ (or $\left(\mathrm{K}^{\prime}\right)_{1}^{3 m}$ ) in $\Omega$. Then $P_{2 m}$ is a hypoelliptic operator with loss of $\frac{2 m(l-1)}{l}$ derivatives.

Lemma 4.14. Assume that the vector fields $\left\{X_{j}\right\}_{j=1}^{k}$ satisfy the condition $(\mathrm{K})_{1}^{\mathrm{d}}$ in $\Omega$. Suppose that $f,\left\{X_{j} f\right\}_{j=1}^{k} \in H_{\text {comp }}^{s_{0}}$ for some real number $s$, where by $H_{\mathrm{comp}}^{s}$ we denote the set of functions in $H^{s}$ having compact support in $\Omega$. Then $X_{\iota} f \in$ $H_{\text {comp }}^{s_{0}-\frac{|\iota|-1}{l}}$ for all multi-orders $\iota$ such that $1<|\iota| \leqslant d$.

Proof. Let $\chi_{\varepsilon} *$ be the averaging operator. In view of the condition $(\mathrm{K})_{1}^{\mathrm{d}}$ we have

$$
\begin{gathered}
\left\|\chi_{\varepsilon} * X_{\iota} f\right\|_{s_{0}-\frac{|\iota|-1}{l}} \leqslant\left\|X_{\iota}\left(\chi_{\varepsilon} * f\right)\right\|_{s_{0}-\frac{|\iota|-1}{l}}+\left\|\left[\chi_{\varepsilon} * X_{\iota}\right] f\right\|_{s_{0}-\frac{|\iota|-1}{l}} \\
\leqslant \sum_{j=1}^{k}\left\|X_{j}\left(\chi_{\varepsilon} * f\right)\right\|_{s_{0}}+C \leqslant \sum_{j=1}^{k}\left(\left\|\chi_{\varepsilon} *\left(X_{j} f\right)\right\|_{s_{0}}+\left\|\left[X_{j}, \chi_{\varepsilon} *\right] f\right\|_{s_{0}}\right)+C \leqslant \tilde{C}
\end{gathered}
$$

for all sufficiently small $\varepsilon$. Tending $\varepsilon \rightarrow 0$ we obtain the desired result.

Lemma 4.15. Assume that the vector fields $\left\{X_{j}\right\}_{j=1}^{k}$ satisfy the conditions $(\mathrm{H})_{1}$ in $\Omega$. Suppose that $f \in \mathcal{E}^{\prime}(\Omega)$ and $\left\{X_{j} f\right\}_{j=1}^{k} \in H_{\mathrm{comp}}^{s_{0}}$ for some real value $s_{0}$. Then $f \in H_{\text {comp }}^{s_{0}+\frac{1}{\tau}}$. 
Proof. Indeed, there exists a number $\sigma$ such that $f \in H_{\text {comp }}^{\sigma}$. Putting $t=\min \left(\sigma, s_{0}\right)$, we have $f,\left\{X_{j} f\right\}_{j=1}^{k} \in H_{\text {comp }}^{t}$. From the condition $(\mathrm{H})_{1}$ it follows that

$$
\left\|\chi_{\varepsilon} * f\right\|_{t+\frac{1}{l}} \leqslant C\left(\sum_{j=1}^{k}\left\|\chi_{\varepsilon} *\left(X_{j} f\right)\right\|_{t}+\sum_{j=1}^{k}\left\|\left[X_{j}, \chi_{\varepsilon} *\right] f\right\|_{t}+\left\|\chi_{\varepsilon} * f\right\|_{t}\right) \leqslant \tilde{C}
$$

for all sufficiently small $\varepsilon$. Hence $f \in H_{\text {comp }}^{t+\frac{1}{\tau}}$. Repeating the argument again and again we come to the needed conclusion.

From Lemmas 4.14 and 4.15, it immediately follows that

Corollary 4.16. Assume that the vector fields $\left\{X_{j}\right\}_{j=1}^{k}$ satisfy the conditions $(\mathrm{H})_{1}$ and $(\mathrm{K})_{1}^{\mathrm{d}}$ in $\Omega$. Suppose that for some real value $s_{0}$ and natural number $p$ the following inclusions hold: $f \in \mathcal{E}^{\prime}(\Omega), X^{\iota} f \in H_{\mathrm{comp}}^{s_{0}-\frac{|\iota|}{l}}$ for all multi-orders $\iota$ such that $1 \leqslant|\iota| \leqslant p$. Then $X_{\iota^{1}} X^{\iota^{2}} f \in H_{\text {comp }}^{s_{0}-\frac{\left|\iota^{1}\right|+\iota^{2} \mid}{l}}$ for all multi-orders $\iota_{1}$ and $\iota_{2}$ such that $\left|\iota^{1}\right| \leqslant d, 0 \leqslant\left|\iota^{2}\right| \leqslant p-1$.

Lemma 4.17. Assume that the vector fields $\left\{X_{j}\right\}_{j=1}^{k}$ satisfy the condition $(\mathrm{H})_{1}$ in $\Omega$. Suppose that $f \in H_{\mathrm{loc}}^{s_{0}}(\Omega),\left\{X_{j} f\right\}_{j=1}^{k} \in H_{\mathrm{loc}}^{s_{0}}(\Omega)$ for some real value $s_{0}$. Then $f \in H_{\mathrm{loc}}^{s_{0}+\frac{1}{l}}(\Omega)$.

Proof. Let $g_{0} \in C_{0}^{\infty}(\Omega)$. We see that

$$
X_{j}\left(g_{0} f\right)=g_{0} X_{j} f+\left[X_{j}, g_{0}\right] f \in H_{\text {comp }}^{s_{0}}, \quad j=\overline{1, k} .
$$

From Lemma 4.15] it follows that $g_{0} f \in H_{\text {comp }}^{s_{0}+\frac{1}{l}}$. Hence $f \in H_{\mathrm{loc}}^{s_{0}+\frac{1}{l}}(\Omega)$ in view of arbitrariness of $g_{0}$. Lemma 4.17 is therefore proved.

Remark 4.18. The assumption $f \in H_{\mathrm{loc}}^{s_{0}}(\Omega)$ in Lemma 4.17 can be replaced by $f \in \mathcal{D}^{\prime}(\Omega)$, or in other words, redundant. In fact, let $\Omega^{\prime} \Subset \Omega$. Then we can find a number $\sigma$ such that $f \in H_{\mathrm{loc}}^{\sigma}\left(\Omega^{\prime}\right)$. Put $t=\min \left\{s_{0}, \sigma\right\}$. It is clear that $f,\left\{X_{j} f\right\}_{j=1}^{k} \in H_{\mathrm{loc}}^{t}\left(\Omega^{\prime}\right)$. Using Lemma 4.17 we obtain $f \in H_{\mathrm{loc}}^{t+\frac{1}{l}}\left(\Omega^{\prime}\right)$. Repeating the above argument again and again we conclude that $f \in H_{\text {loc }}^{s_{0}+\frac{1}{l}}\left(\Omega^{\prime}\right)$. In view of the arbitrariness of $\Omega^{\prime}$ we arrive at $f \in H_{\mathrm{loc}}^{t+\frac{1}{l}}(\Omega)$.

Corollary 4.19. Assume that the vector fields $\left\{X_{j}\right\}_{j=1}^{k}$ satisfy the condition $(\mathrm{H})_{1}$ in $\Omega$. If $X^{\iota} f \in H_{\mathrm{loc}}^{s_{0}}(\Omega)$ for some real value $s_{0}$ and all multi-orders $\iota$ such that $|\iota| \leqslant p$, then $X^{\iota} f \in H_{\mathrm{loc}}^{s_{0}+\frac{p-|\iota|}{l}}(\Omega)$ for all multi-orders $\iota$ such that $|\iota| \leqslant p-1$.

Lemma 4.20. Assume that the vector fields $\left\{X_{j}\right\}_{j=1}^{k}$ satisfy the conditions $(\mathrm{H})_{1}$ and $(\mathrm{K})_{1}^{\mathrm{d}}$ in $\Omega$. Suppose that for some real value $s_{0}$ and natural integer $p$ the inclusions $X^{\iota} f \in H_{\mathrm{loc}}^{s_{0}-\frac{|\iota|}{l}}(\Omega)$ hold for all multi-orders $\iota$ such that $1 \leqslant|\iota| \leqslant p$. Then $X_{\iota^{1}} X^{\iota^{2}} f \in H_{\mathrm{loc}}^{s_{0}-\frac{\iota^{1}|+| \iota^{2} \mid}{l}}(\Omega)$ for all multi-orders $\iota^{1}, \iota^{2}$ such that $\left|\iota^{1}\right| \leqslant d, 0 \leqslant\left|\iota^{2}\right| \leqslant$ $p-1$.

Proof. Let $g_{0} \in C_{0}^{\infty}(\Omega)$ as in Lemma 4.17, Then

$$
X_{j}\left(g_{0} X^{\iota^{2}} f\right)=g_{0} X_{j} X^{\iota^{2}} f+\left[X_{j}, g_{0}\right] X^{\iota^{2}} f \in H_{\text {comp }}^{s_{0}-\frac{\iota^{2} \mid+1}{l}}, j=1, \ldots, k .
$$


By Lemma 4.14 we have $X_{\iota^{1}}\left(g_{0} X^{\iota^{2}} f\right) \in H_{\text {comp }}^{s_{0}-\frac{\iota^{1}|+| \iota^{2} \mid}{l}}$. Hence

$$
g_{0} X_{\iota^{1}} X^{\iota^{2}} f=X_{\iota^{1}}\left(g_{0} X^{\iota^{2}} f\right)+\left[g_{0}, X_{\iota^{1}}\right] X^{\iota^{2}} f \in H_{\text {comp }}^{s_{0}-\frac{\left|\iota^{1}\right|+\left|\iota^{2}\right|}{l}} .
$$

In view of the arbitrariness of $g_{0}$ we deduce that $X_{\iota} X^{\iota^{2}} f \in H_{\mathrm{loc}}^{s_{0}-\frac{\left|\iota^{1}\right|+\left|\iota^{2}\right|}{l}}(\Omega)$. Lemma 4.20 is therefore proved.

Proposition 4.21. Assume that the vector fields $\left\{X_{j}\right\}_{j=1}^{k}$ satisfy the conditions $(\mathrm{H})_{1}$ and $(\mathrm{K})_{1}^{\mathrm{d}}$ in $\Omega$, and $Q_{m}$ is (see the formula (2.1) ) maximally $\frac{1}{l}$-hypoelliptic. Then from $f \in \mathcal{D}^{\prime}(\Omega), Q_{m} f \in H_{\mathrm{loc}}^{s}(\Omega)$ it follows that $X^{\iota} f \in H_{\mathrm{loc}}^{s+\frac{m-|\iota|}{l}}(\Omega)$ for all multi-orders $\iota$ such that $0 \leqslant|\iota| \leqslant m$ and $X_{\iota^{1}} X^{\iota^{2}} f \in H_{\mathrm{loc}}^{s+\frac{m-\left|\iota^{1}\right|-\left|\iota^{2}\right|}{l}}(\Omega)$ for all multi-orders $\iota^{1}, \iota^{2}$ such that $\left|\iota^{1}\right| \leqslant d, 0 \leqslant\left|\iota^{2}\right| \leqslant m-1$.

Proof. From the definition of maximal $\frac{1}{l}$-hypoellipticity it follows that $X^{\iota} f \in$ $H_{\text {loc }}^{s+\frac{m-|\iota|}{l}}(\Omega)$ for all multi-orders $\iota$ such that $0 \leqslant|\iota| \leqslant m$. The proof of this fact is similar to that for Lemma 4.14. Now the last statement of Proposition 4.21 follows directly from Lemma 4.20 .

Theorem 4.22. Assume that the vector fields $\left\{X_{j}\right\}_{j=1}^{k}$ satisfy the conditions $(\mathrm{H})_{1}$ and $(\mathrm{K})_{1}$ in $\Omega$. Then $P_{2 m}$ is an extendedly maximally hypoelliptic operator in the system $\left\{X_{j}\right\}_{j=1}^{k}$.

Proof. We prove that if $P_{2 m} f \in S_{\mathrm{loc}}^{q,\left\{X_{j}\right\}_{j=1}^{k}}(\Omega)$, then $f \in S_{\mathrm{loc}}^{q+2 m,\left\{X_{j}\right\}_{j=1}^{k}}(\Omega)$. To this end we establish that $P_{2 m}\left(X^{\iota} f\right) \in H_{\mathrm{loc}}^{\frac{q-|\iota|}{c}}(\Omega)$ by induction on $\iota$ with $0 \leqslant|\iota| \leqslant q$. For $|\iota|=0$ the claim is trivial since $P_{2 m} f \in S_{\text {loc }}^{q,\left\{X_{j}\right\}_{j=1}^{k}}(\Omega) \subset H_{\mathrm{loc}}^{\frac{q}{q}}(\Omega)$. Assuming that our claim is true for $|\iota|=q_{0} \leqslant q-1$, we prove it for all multi-orders $\iota$ such that $|\iota|=q_{0}+1$. From the inductive assumption we deduce that $X^{\vartheta} f \in H_{\mathrm{loc}}^{\frac{q+2 m-|\vartheta|}{l}}(\Omega)$ for all multi-orders $\vartheta$ such that $|\vartheta| \leqslant 2 m+q_{0}$. On the other hand, for all multi-orders $\iota$ with $|\iota|=q_{0}+1$ we have

$$
P_{2 m}\left(X^{\iota} f\right)=X^{\iota}\left(P_{2 m} f\right)+\sum_{\substack{\left|\iota^{1}\right|+\left|\iota^{2}\right| \leqslant 2 m+q_{0}+1 \\\left|\iota^{2}\right| \leqslant 2 m+q_{0}-1}} a_{\iota^{1}, \iota^{2}} X_{\iota^{1}} X^{\iota^{2}} f .
$$

Hence by Lemma 4.20, we conclude that $P_{2 m}\left(X^{\iota} f\right) \in H_{1 \mathrm{loc}}^{\frac{q-\left(q_{0}+1\right)}{l}}(\Omega)$, which is exactly the claim for $|\iota|=q_{0}+1$. Now for $|\iota|=q$ we have $P_{2 m}\left(X^{\iota} f\right) \in L_{\text {loc }}^{2}(\Omega)$. From the maximal $\frac{1}{l}$-hypoellipticity of $P_{2 m}$ we deduce that $X^{\vartheta} f \in L_{\text {loc }}^{2}(\Omega)$ for all multi-orders $\vartheta$ with $|\vartheta| \leqslant 2 m+q$. The proof of Theorem 4.22 is therefore complete.

Remark 4.23. From the microlocal point of view the condition $(\mathrm{H})_{1}$ can be formulated for a system $\left\{X_{j}\right\}_{j=1}^{k}$, consisting of proper first order p.d.o. with real principal symbols. Theorems analogous to Theorems 4.10- 4.22 remain valid for operators of the form

$$
P_{2 m}^{\prime}=\sum_{j=1}^{k} A_{j}(x, \partial) X_{j}^{2 m}+\sum_{|\iota| \leqslant 2 m-1} B_{\iota}(x, \partial) X^{\iota},
$$

where $A_{j}(x, \partial), B_{\iota}(x, \partial)$ are proper zero order p.d.o. Furthermore, $A_{j}(x, \partial)$ are supposed to be elliptic and positive operators. 
In the particular case when the condition $(\mathrm{H})_{2}$ is satisfied, the maximal $\frac{1}{2}$ hypoellipticity of the operator $P_{2 m}^{\prime}$ was proved in [4, 5]. In this particular case, from the condition $(\mathrm{H})_{2}$ alone we can deduce the condition $(\mathrm{K})_{2}$. As was pointed out in [5], the condition $(\mathrm{H})_{2}$ follows from the noninvolutiveness of the characteristic set of $P_{2 m}^{\prime}$ in the cotangent space.

4.1.2. Some examples. In practice it may not be easy to verify the conditions $(\mathrm{K})_{1}^{\mathrm{d}}$ and $\left(\mathrm{K}^{\prime}\right)_{1}^{\mathrm{d}}$. In this part we give some examples of systems that satisfy the condition $(\mathrm{K})_{1}^{\mathrm{d}}$.

a) Complete system of vector fields degenerate on a submanifold. Let the variable $x \in \mathbb{R}^{n}$ be separated into two groups $x=\left(x^{\prime}, y\right)$, where $x^{\prime}=\left(x_{1}, \ldots, x_{n_{1}}\right)$, $y=\left(y_{1}, \ldots, y_{n_{2}}\right), n_{1}+n_{2}=n$. Consider the following system of vector fields:

$$
\begin{gathered}
X_{1}=\frac{\partial}{\partial x_{1}}, \ldots, X_{n_{1}}=\frac{\partial}{\partial x_{n_{1}}}, X_{1,1}=x_{1}^{l} \frac{\partial}{\partial y_{1}}, \ldots, X_{1, n_{2}}=x_{1}^{l} \frac{\partial}{\partial y_{n_{2}}}, \ldots, \\
X_{n_{1}, 1}=x_{n_{1}}^{l} \frac{\partial}{\partial y_{1}}, \ldots, X_{n_{1}, n_{2}}=x_{n_{1}}^{l} \frac{\partial}{\partial y_{n_{2}}} .
\end{gathered}
$$

Clearly, the system $X_{1}, \ldots, X_{n_{1}}, X_{1,1}, \ldots, X_{1, n_{2}}, \ldots, X_{n_{1}, 1}, \ldots, X_{n_{1}, n_{2}}$ satisfies the condition $(\mathrm{H})_{1+1}$. Now we show that it satisfies the condition $(\mathrm{K})_{1+1}$, too. Indeed, only the commutators $\left[{ }_{i} X_{j}, X_{j, j^{\prime}}\right], 1 \leqslant i \leqslant l, j=\overline{1, n_{1}}, j^{\prime}=\overline{1, n_{2}}$, differ from zero. We will estimate these commutators on the space $C_{0}^{\infty}(K)$, where $K$ is a fixed compact in $\Omega$. By using the Cauchy - Schwarz inequality, for any two real values $s, s_{1}$ with $s-1 \leqslant s_{1}$ and all $\varepsilon>0$ there exists a constant $C(\varepsilon)$ such that

$$
\left\|x_{1}^{i-1} \frac{\partial f}{\partial y_{1}}\right\|_{s} \leqslant \varepsilon\left\|x_{1}^{i} \frac{\partial f}{\partial y_{1}}\right\|_{2 s-s_{1}}+C(\varepsilon)\left\|x_{1}^{i-2} \frac{\partial f}{\partial y_{1}}\right\|_{s_{1}}, 2 \leqslant i .
$$

Hence,

$$
\left\|x_{1}^{l-1} \frac{\partial f}{\partial y_{1}}\right\|_{0} \leqslant \varepsilon\left\|x_{1}^{l} \frac{\partial f}{\partial y_{1}}\right\|_{\frac{1}{l+1}}+C_{1}(\varepsilon)\left\|x_{1}^{l-2} \frac{\partial f}{\partial y_{1}}\right\|_{-\frac{1}{l+1}}, 2 \leqslant l .
$$

From $C_{1}(\varepsilon)$ we can find a constant $C_{2}(\varepsilon)$ such that

$$
\left(2+C_{1}(\varepsilon)\right)\left\|x_{1}^{l-2} \frac{\partial f}{\partial y_{1}}\right\|_{-\frac{1}{l+1}} \leqslant\left\|x_{1}^{l-1} \frac{\partial f}{\partial y_{1}}\right\|_{0}+C_{2}(\varepsilon)\left\|x_{1}^{l-3} \frac{\partial f}{\partial y_{1}}\right\|_{-\frac{2}{l+1}} .
$$

Continuing the process, on the $i$-th $(i \leqslant l-1)$ step we obtain

$$
\left(2+C_{i-1}(\varepsilon)\right)\left\|x_{1}^{l-i} \frac{\partial f}{\partial y_{1}}\right\|_{-\frac{i-1}{l+1}} \leqslant\left\|x_{1}^{l-i+1} \frac{\partial f}{\partial y_{1}}\right\|_{-\frac{i-2}{l+1}}+C_{i}(\varepsilon)\left\|x_{1}^{l-i-1} \frac{\partial f}{\partial y_{1}}\right\|_{-\frac{i}{l+1}} .
$$

The process will finish on the $(l-1)$-th step, on which we have

$$
\left(2+C_{l-2}(\varepsilon)\right)\left\|x_{1} \frac{\partial f}{\partial y_{1}}\right\|_{-\frac{l-2}{l+1}} \leqslant\left\|x_{1}^{2} \frac{\partial f}{\partial y_{1}}\right\|_{-\frac{l-3}{l+1}}+C_{l-1}(\varepsilon)\left\|\frac{\partial f}{\partial y_{1}}\right\|_{-\frac{l-1}{l+1}} .
$$

Now applying the estimate for commutators

$$
\|[X, Y] f\|_{s} \leqslant \varepsilon\left(\|X f\|_{s_{1}}+\|f\|_{s_{1}}\right)+C(\varepsilon)\left(\|Y f\|_{1+2 s-s_{1}}+\|f\|_{1+2 s-s_{1}}\right), \forall s, s_{1} \in \mathbb{R},
$$

to $\frac{\partial}{\partial y_{1}}=\left[\frac{\partial}{\partial x_{1}}, x_{1} \frac{\partial}{\partial y_{1}}\right]$, we conclude that from $C_{l-1}(\varepsilon)$ there can be found a constant $C_{l}(\varepsilon)$ such that

$$
\left(2 n_{1} n_{2}+C_{l-1}(\varepsilon)\right)\left\|\frac{\partial f}{\partial y_{1}}\right\|_{-\frac{l-1}{l+1}} \leqslant\left\|x_{1} \frac{\partial f}{\partial y_{1}}\right\|_{-\frac{l-2}{l+1}}+C_{l}(\varepsilon)\left(\left\|\frac{\partial f}{\partial x_{1}}\right\|_{\frac{1}{l+1}}+\|f\|_{\frac{1}{l+1}}\right) .
$$


On the other hand, based on the two well-known inequalities

$$
2 C\|f\|_{\frac{2}{l+1}} \leqslant \sum_{j=1}^{n_{1}}\left\|\frac{\partial f}{\partial x_{j}}\right\|_{\frac{1}{l+1}}+\sum_{j=1}^{n_{2}}\left\|\frac{\partial f}{\partial y_{j}}\right\|_{-\frac{l-1}{l+1}}+\|f\|_{0},
$$

where the constant $C$ does not depend on $C_{1}(\varepsilon), \ldots, C_{l}(\varepsilon)$, and

$$
C(\varepsilon)\|f\|_{\frac{1}{l+1}} \leqslant C\|f\|_{\frac{2}{l+1}}+\tilde{C}(\varepsilon)\|f\|_{0},
$$

from $C_{l}(\varepsilon)$ one can find $C_{l+1}(\varepsilon)$ such that

$$
\left(2+C_{l}(\varepsilon)\right)\|f\|_{\frac{1}{l+1}}+C\|f\|_{\frac{2}{l+1}} \leqslant \sum_{j=1}^{n_{1}}\left\|\frac{\partial f}{\partial x_{j}}\right\|_{\frac{1}{l+1}}+\sum_{j=1}^{n_{2}}\left\|\frac{\partial f}{\partial y_{j}}\right\|_{-\frac{l-1}{l+1}}+C_{l+1}(\varepsilon)\|f\|_{0} .
$$

By the same trick we can obtain similar estimates for $x_{j} \frac{\partial f}{\partial y_{j^{\prime}}}, j=\overline{1, n_{1}}, j^{\prime}=\overline{1, n_{2}}$. Summing up all the obtained inequalities, then reducing similar terms in both sides, one can find

$$
\begin{gathered}
\max \left\{\|f\|_{\frac{2}{l+1}},\left\|x_{j}^{l-i} \frac{\partial f}{\partial y_{j^{\prime}}}\right\|_{-\frac{i-1}{l+1}}\left(j=\overline{1, n_{1}}, j^{\prime}=\overline{1, n_{2}}, 1 \leqslant i \leqslant l\right)\right\} \\
\leqslant \varepsilon \sum_{j=1}^{n_{1}} \sum_{j^{\prime}=1}^{n_{2}}\left\|x_{j}^{l} \frac{\partial f}{\partial y_{j^{\prime}}}\right\|_{\frac{1}{l+1}}+C(\varepsilon)\left(\sum_{j=1}^{n_{1}}\left\|\frac{\partial f}{\partial x_{j}}\right\|_{\frac{1}{l+1}}+\|f\|_{0}\right) .
\end{gathered}
$$

Since all the operators in the above estimate have order 1 , by standard arguments we deduce

$$
\begin{aligned}
& \max \left\{\|f\|_{s+\frac{2}{l+1}},\left\|x_{j}^{l-i} \frac{\partial f}{\partial y_{j^{\prime}}}\right\|_{s-\frac{i-1}{l+1}}\left(j=\overline{1, n_{1}}, j^{\prime}=\overline{1, n_{2}}, 1 \leqslant i \leqslant l\right)\right\} \\
& \leqslant \varepsilon \sum_{j=1}^{n_{1}} \sum_{j^{\prime}=1}^{n_{2}}\left\|x_{j}^{l} \frac{\partial f}{\partial y_{j^{\prime}}}\right\|_{s+\frac{1}{l+1}}+C(\varepsilon)\left(\sum_{j=1}^{n_{1}}\left\|\frac{\partial f}{\partial x_{j}}\right\|_{s+\frac{1}{l+1}}+\|f\|_{0}\right), \forall s \in \mathbb{R} .
\end{aligned}
$$

If we take into account the formula $x_{1}^{l-i} \frac{\partial f}{\partial y_{1}} \approx\left[i \frac{\partial f}{\partial x_{1}}, x_{1}^{l} \frac{\partial f}{\partial y_{1}}\right]$, by choosing $\varepsilon=1$, we arrive at the desired result. Thus, the system $X_{1}, \ldots, X_{n_{1}}, X_{1,1}, \ldots, X_{1, n_{2}}, \ldots$, $X_{n_{1}, 1}, \ldots, X_{n_{1}, n_{2}}$ satisfies the condition $(\mathrm{K})_{1+1}$. Therefore, according to Subsubsection 4.1.1] we can formulate a theorem on the maximal $\frac{1}{l+1}$-hypoellipticity of the operator $P_{2 m}$ consisting of the vector fields from the system. Here we will not do it, leaving the details to the readers. But we note that the estimate (4.11), in fact, is stronger than the ones required in the condition $(\mathrm{K})_{1+1}$.

b) Noncomplete system of vector fields degenerate on a submanifold. Let the variable $x \in \mathbb{R}^{n}$ be separated into three groups $x=\left(x^{\prime}, y, z\right)$, where $x^{\prime}=\left(x_{1}, \ldots, x_{n_{1}}\right)$, $y=\left(y_{1}, \ldots, y_{n_{2}}\right), z=\left(z_{1}, \ldots, z_{n_{3}}\right), n_{1}+n_{2}+n_{3}=n$. Consider the following system of vector fields:

$$
\begin{aligned}
& X_{1}=\frac{\partial}{\partial x_{1}}, \ldots, X_{n_{1}}=\frac{\partial}{\partial x_{n_{1}}}, X_{1,1}=x_{1}^{l} \frac{\partial}{\partial y_{1}}, \ldots, X_{1, n_{2}}=x_{1}^{l} \frac{\partial}{\partial y_{n_{2}}}, \ldots, \\
& X_{n_{1}, 1}=x_{n_{1}}^{l} \frac{\partial}{\partial y_{1}}, \ldots, X_{n_{1}, n_{2}}=x_{n_{1}}^{l} \frac{\partial}{\partial y_{n_{2}}}, Z_{1}=\frac{\partial}{\partial z_{1}}, \ldots, Z_{n_{3}}=\frac{\partial}{\partial z_{n_{3}}} .
\end{aligned}
$$

Again the system $X_{1}, \ldots, X_{n_{1}}, X_{1,1}, \ldots, X_{1, n_{2}}, \ldots, X_{n_{1}, 1}, \ldots, X_{n_{1}, n_{2}}, Z_{1}, \ldots, Z_{n_{3}}$ satisfies the condition $(\mathrm{H})_{1+1}$. Also, in this case one can show that this system 
satisfies the condition $(\mathrm{K})_{1+1}$, too. Indeed, all the arguments used in the previous example can be applied here. Only one estimate (4.10) needs to be modified by

$$
2 C\|f\|_{\frac{2}{l+1}} \leqslant \sum_{j=1}^{n_{1}}\left\|\frac{\partial f}{\partial x_{j}}\right\|_{\frac{1}{l+1}}+\sum_{j=1}^{n_{2}}\left\|\frac{\partial f}{\partial y_{j}}\right\|_{-\frac{l-1}{l+1}}+\sum_{j=1}^{n_{3}}\left\|\frac{\partial f}{\partial z_{j}}\right\|_{-\frac{l-1}{l+1}}+\|f\|_{0} .
$$

Hence from $C_{l}(\varepsilon)$ one can find $C_{l+1}(\varepsilon)$ such that

$$
\begin{aligned}
& \left(2+C_{l}(\varepsilon)\right)\|f\|_{\frac{1}{l+1}}+C\|f\|_{\frac{2}{l+1}} \\
& \leqslant \sum_{j=1}^{n_{1}}\left\|\frac{\partial f}{\partial x_{j}}\right\|_{\frac{1}{l+1}}+\sum_{j=1}^{n_{2}}\left\|\frac{\partial f}{\partial y_{j}}\right\|_{-\frac{l-1}{l+1}}+\sum_{j=1}^{n_{3}}\left\|\frac{\partial f}{\partial z_{j}}\right\|_{-\frac{l-1}{l+1}}+C_{l+1}(\varepsilon)\|f\|_{0} .
\end{aligned}
$$

Finally we can establish the following estimates for all $s \in \mathbb{R}$ :

$$
\begin{gathered}
\max \left\{\|f\|_{s+\frac{2}{l+1}},\left\|x_{j}^{l-i} \frac{\partial f}{\partial y_{j^{\prime}}}\right\|_{s-\frac{i-1}{l+1}}\left(j=\overline{1, n_{1}}, j^{\prime}=\overline{1, n_{2}}, 1 \leqslant i \leqslant l\right)\right\} \\
\leqslant \varepsilon \sum_{j=1}^{n_{1}} \sum_{j^{\prime}=1}^{n_{2}}\left\|x_{j}^{l} \frac{\partial f}{\partial y_{j^{\prime}}}\right\|_{s+\frac{1}{l+1}}+C \sum_{j=1}^{n_{3}}\left\|\frac{\partial f}{\partial z_{j}}\right\|_{s-\frac{l-1}{l+1}}+C(\varepsilon)\left(\sum_{j=1}^{n_{1}}\left\|\frac{\partial f}{\partial x_{j}}\right\|_{s+\frac{1}{l+1}}+\|f\|_{0}\right) .
\end{gathered}
$$

Note that the estimates we get here in the variables $z_{1}, \ldots, z_{n_{3}}$ are better than what is required in the condition $(\mathrm{K})_{l+1}$. Again a theorem on the maximal $\frac{1}{l+1}$ hypoellipticity can be formulated for the operator $P_{2 m}$ constructing from the vector fields $X_{1}, \ldots, X_{n_{1}}, X_{1,1}, \ldots, X_{1, n_{2}}, \ldots, X_{n_{1}, 1}, \ldots, X_{n_{1}, n_{2}}, Z_{1}, \ldots, Z_{n_{3}}$. We omit the details.

4.1.3. Semilinear equations. In this part we study a semilinear perturbation of the operator $P_{2 m}$ considered in Subsubsection 4.1.1

$$
\Psi_{2 m}\left(x, \partial^{\alpha}\right): f \longrightarrow P_{2 m}(x, D) f+\Phi\left(x, X^{\iota} f\right)_{|\iota| \leqslant 2 m-1} .
$$

First we establish a theorem of general character for the nonlinear operator $\Theta_{m}$ given by the formula (2.3).

Theorem 4.24. Let $Q_{m}$ be extendedly maximally hypoelliptic in the system $\left\{X_{j}\right\}_{j=1}^{k}$ and the function $\Phi\left(x, \tau_{\iota}\right)_{|\iota| \leqslant m-1}$ is infinitely differentiable in its variables. Suppose that the system $\left\{X_{j}\right\}_{j=1}^{k}$ satisfies the smoothness condition. Then the nonlinear operator $\Theta_{m}$ is extendedly maximally hypoelliptic in the system $\left\{X_{j}\right\}_{j=1}^{k}$. In particular, it is hypoelliptic.

Proof. In view of the smoothness condition of the system $\left\{X_{j}\right\}_{j=1}^{k}$ and the Sobolev embedding theorem one can find a number $M$ such that $S_{\text {loc }}^{M,\left\{X_{j}\right\}_{j=1}^{k}}(\Omega) \subset C(\Omega)$. Now supposing that $m_{1} \geq 2 M+m$ we can repeat the arguments used in Proposition 3.2 for getting the following claim: if $f \in S_{\mathrm{loc}}^{m_{1},\left\{X_{j}\right\}_{j=1}^{k}}(\Omega)$, then $\Phi\left(x, X^{\iota} f\right)_{|\iota| \leqslant m-1} \in$ $S_{\text {loc }}^{m_{1}-(m-1),\left\{X_{j}\right\}_{j=1}^{k}}(\Omega)$. Further, based on the claim we just obtained, we can apply the technique used in the proof of Theorem 3.3 to get the desired result.

From Theorems 4.22 and 4.24 we get

Theorem 4.25. Let the vector fields $\left\{X_{j}\right\}_{j=1}^{k}$ satisfy the conditions $(\mathrm{H})_{1}$ and $(\mathrm{K})_{1}$ (or $\left.\left(\mathrm{K}^{\prime}\right)_{1}\right)$ in $\Omega$. If the function $\Phi\left(x, \tau_{\iota}\right)_{|\iota| \leqslant 2 m-1}$ is infinitely differentiable in its variables, then the nonlinear operator $\Psi_{2 m}$ is hypoelliptic. Furthermore, it is extendedly maximally hypoelliptic in the system $\left\{X_{j}\right\}_{j=1}^{k}$. 
From the results in Subsubsection 4.1.2 of this section, based on Theorem 4.25 we give two examples

Example 4.26. The nonlinear operator

$$
\Psi_{2 m}^{(1)}(f)=\frac{\partial^{2 m} f}{\partial x^{2 m}}+x^{2 m k}\left(\frac{\partial^{2 m} f}{\partial y^{2 m}}+\frac{\partial^{2 m} f}{\partial z^{2 m}}\right)+\cos \left(x^{(2 m-1) k} \frac{\partial^{2 m-1} f}{\partial y^{2 m-1}}\right) \mathrm{e}^{\frac{\partial^{2 m-1} f}{\partial x^{2 m-1}}}
$$

is hypoelliptic in $\mathbb{R}^{3}$.

Example 4.27. The nonlinear operator

$$
\Psi_{2 m}^{(2)}(f)=\frac{\partial^{2 m} f}{\partial x^{2 m}}+x^{2 m k} \frac{\partial^{2 m} f}{\partial y^{2 m}}+\frac{\partial^{2 m} f}{\partial z^{2 m}}+\left(\frac{\partial^{2 m-1} f}{\partial x^{2 m-1}}\right)^{5}\left(x^{k} \frac{\partial f}{\partial y}\right)^{2}
$$

is hypoelliptic in $\mathbb{R}^{3}$.

4.2. Maximal $\delta$-hypoellipticity without condition $(\mathbf{K})_{1}$. In this part we investigate hypoellipticity of the semilinear operator $\Psi_{4}$, however, without the condition $(\mathrm{K})_{1}$. We will prove theorems on the weakly maximal $\delta$-hypoellipticity of the operator $P_{4}$, then its maximal $\delta$-hypoellipticity, and finally the extendedly maximal hypoellipticity of its semilinear perturbation. First, we establish a theorem of general character for $Q_{m}$.

Lemma 4.28. Let $Q_{m}$ be weakly maximally $\delta$-hypoelliptic in $\Omega$. Assume that $X^{\iota} f, Q_{m} f \in H_{\text {comp }}^{s}(0 \leqslant|\iota| \leqslant m-1)$ for some real number s. Then $X^{\iota} f \in$ $H_{\text {comp }}^{s+\delta(m-|\iota|)}$ for $0 \leqslant|\iota| \leqslant m-1$.

Proof. We prove Lemma 4.28 by induction on $|\iota|$. In view of the weakly maximal $\delta$-hypoellipticity of $Q_{m}$ we have

$$
\left\|\chi_{\varepsilon} * f\right\|_{s+\delta m} \leqslant C\left(\left\|Q_{m}\left(\chi_{\varepsilon} * f\right)\right\|_{s}+\left\|\chi_{\varepsilon} * f\right\|_{s}\right) \leqslant C\left(\left\|\chi_{\varepsilon} * Q_{m} f\right\|_{s}+\left\|\chi_{\varepsilon} * f\right\|_{s}\right.
$$

$$
\left.+\sum_{|\iota| \leqslant m}\left\|\left[a_{\iota}, \chi_{\varepsilon} *\right] X^{\iota} f\right\|_{s}+\sum_{|\iota| \leqslant m} \sum_{\iota^{1} \subseteq \iota, \iota^{1} \neq \iota}\left\|\left[\iota \iota^{1} X, \chi_{\varepsilon} *\right] X^{\iota^{1}} f\right\|_{s}\right):=I .
$$

By the assumptions of Lemma 4.28 the right hand side of (4.12) is bounded (uniformly in $\varepsilon$ ). Hence, tending $\varepsilon \rightarrow 0$ we conclude that $f \in H_{\text {comp }}^{s+\delta m}$. Thus, Lemma 4.28 is proved for $|\iota|=0$. Assuming that Lemma 4.28 is true for $|\iota| \leqslant p \leqslant m-2$, we prove it for $|\iota|=p+1$. Again in view of the maximal $\delta$-hypoellipticity of $Q_{m}$ the following inequality holds:

$$
\left\|X^{\iota}\left(\chi_{\varepsilon} * f\right)\right\|_{s+\delta(m-p-1)} \leqslant I \text { for }|\iota|=p+1 .
$$

On the other hand,

$$
\begin{aligned}
\left\|\chi_{\varepsilon} * X^{\iota} f\right\|_{s+\delta(m-p-1)} & \leqslant\left\|X^{\iota}\left(\chi_{\varepsilon} * f\right)\right\|_{s+\delta(m-p-1)} \\
& +\sum_{\iota^{1} \subseteq \iota, \iota^{1} \neq \iota}\left\|\left[\iota \iota^{1} X, \chi_{\varepsilon} *\right] X^{\iota^{1}} f\right\|_{s+\delta(m-p-1)} \leqslant I+J .
\end{aligned}
$$

By the inductive assumptions, $|J|$ is bounded. Therefore, $\left\|\chi_{\varepsilon} * X^{\iota} f\right\|_{s+\delta(m-p-1)}$ is bounded. Hence $X^{\iota} f \in H_{\text {comp }}^{s+\delta(m-p-1)}$ for $|\iota|=p+1$. Lemma 4.28 is proved.

Corollary 4.29. Let $Q_{m}$ be weakly maximally $\delta$-hypoelliptic in $\Omega$. If $f \in \mathcal{E}^{\prime}(\Omega)$, $Q_{m} f \in H^{s}$ for some real number $s$, then $X^{\iota} f \in H^{s+\delta(m-|\iota|)}$ for $0 \leqslant|\iota| \leqslant m-1$. 
Proof. Since $f \in \mathcal{E}^{\prime}(\Omega)$ there exists a number $\sigma$ such that $X^{\iota} f \in H^{\sigma}$ for $|\iota| \leqslant m-1$. Put $t=\min (s, \sigma)$. Applying Lemma4.28 we get $X^{\iota} f \in H^{t+\delta(m-|\iota|)}$. We can repeat the argument again and again with $\min (s, t+\delta)$ instead of $t$ and obtain the analogous claim. After a number of steps we arrive at $X^{\iota} f \in H^{s}$ for $|\iota| \leqslant m-1$. Again using Lemma 4.28 we deduce the desired result.

Theorem 4.30. Let $Q_{m}$ be weakly maximally $\delta$-hypoelliptic in $\Omega$. Then $Q_{m}$ is hypoelliptic in $\Omega$.

Proof. The proof of Theorem 4.30 is based on a standard procedure and Corollary 4.29. We give it for completeness. We will prove that if $s \in \mathbb{R}$ and $Q_{m} f \in H_{\text {loc }}^{s}(\Omega)$, then

$$
X^{\iota} f \in H_{\mathrm{loc}}^{s+\delta(m-|\iota|)}(\Omega), 0 \leqslant|\iota| \leqslant m-1 .
$$

Let $\Omega^{\prime} \Subset \Omega$ and $f \in \mathcal{D}^{\prime}(\Omega)$. Then $f \in H_{\text {loc }}^{\sigma}\left(\Omega^{\prime}\right)$ for some $\sigma \in \mathbb{R}$. We will use the following notation from [15]: for two functions $a, b \in C_{0}^{\infty}(\Omega)$ we write $a \lesssim b$ if $b \equiv 1$ on the support $a$. Take a sequence of $C^{\infty}$-functions $\left\{g_{i}\right\}_{i=0}^{\infty}$ such that $g_{i} \lesssim g_{i+1}$. Obviously,

$$
Q_{m}\left(g_{i} f\right)=g_{i} Q_{m} f+\sum_{\iota^{1} \subseteq \iota, \iota^{1} \neq \iota} a_{\iota}\left[\iota \iota^{1} X, g_{i}\right] X^{\iota^{1}}\left(g_{i+1} f\right) .
$$

Put $t=\min (s, \sigma)$. Then the right hand side of (4.13) belongs to $H^{t-m+1}$, i.e. $Q_{m}\left(g_{i} f\right) \in H^{t-m+1}$. Hence, by Corollary 4.29

$$
X^{\iota}\left(g_{i} f\right) \in H^{t-m+1+\delta(m-|\iota|)} \quad(0 \leqslant|\iota| \leqslant m-1) .
$$

Thus, the right hand side of (4.13) belongs to $H^{t-m+1+\delta}$. Therefore

$$
X^{\iota}\left(g_{i-1} f\right) \in H^{t-(m-1)+\delta(m+1-|\iota|)} \quad(0 \leqslant|\iota| \leqslant m-1) .
$$

Repeating the argument again a finite number of times we obtain $Q_{m}\left(g_{0} f\right) \in H^{t}$. Hence

$$
X^{\iota}\left(g_{0} f\right) \in H^{t+\delta(m-|\iota|)} \quad(0 \leqslant|\iota| \leqslant m-1) .
$$

Thus, we have proved

$$
\begin{aligned}
f \in H_{\mathrm{loc}}^{t}(\Omega), Q_{m} f \in H_{\mathrm{loc}}^{t}(\Omega) & \Rightarrow f \in H_{\mathrm{loc}}^{t+\delta m}\left(\Omega^{\prime}\right), X^{\iota} f \in H_{\mathrm{loc}}^{t+\delta(m-|\iota|)}\left(\Omega^{\prime}\right), \\
0 & \leqslant|\iota| \leqslant m-1 .
\end{aligned}
$$

Again and again using the statement just obtained we come to $t=s$, and in view of arbitrariness of $\Omega^{\prime}$ we get the desired result.

Lemma 4.31. Let the vector fields $\left\{X_{j}\right\}_{j=1}^{k}$ satisfy the condition $(\mathrm{H})_{3}$ in $\Omega$. Then for any compact $K$ in $\Omega$, and each real value $s$ there exists a constant $C(s, K)=C$ such that

$$
\left\|X^{\iota} f\right\|_{s}^{2} \leqslant C\left(\sum_{j=1}^{k}\left\|X_{j}^{3} f\right\|_{s+\frac{1}{6}}^{2}+\|f\|_{s}^{2}\right)(|\iota|=3), \quad \forall f \in C_{0}^{\infty}(K) .
$$

Proof. Denote the right hand side of (4.14) by $I_{s}$. Note that we can write $\iota=\iota^{1}+\iota^{2}$ with $\left|\iota^{1}\right|=2,\left|\iota^{2}\right|=1$. By Theorem 2.4

$$
\left\|X^{\iota} f\right\|_{s}^{2}=\left\|X^{\iota^{1}}\left(X^{\iota^{2}} f\right)\right\|_{s}^{2} \leqslant C\left(\sum_{j=1}^{k}\left\|X_{j}^{2}\left(X^{\iota^{2}} f\right)\right\|_{s}^{2}+\|f\|_{s}^{2}\right) .
$$


Thus, the matter reduces to estimating the terms of the form $\left\|X_{j_{1}}^{2} X_{j_{2}} f\right\|_{s}^{2}, j_{1} \neq j_{2}$. We have

$$
\begin{aligned}
\left\|X_{j_{1}}^{2} X_{j_{2}} f\right\|_{0}^{2} & =\left(X_{j_{1}}^{2} X_{j_{2}} f, X_{j_{1}}^{2} X_{j_{2}} f\right)_{0} \leqslant\left|\left(X_{j_{1}} X_{j_{2}} f, X_{j_{2}} X_{j_{1}}^{3} f\right)_{0}\right| \\
& +\sum_{i=1}^{3} C_{3}^{i}\left|\left(X_{j_{1}} X_{j_{2}} f,\left[{ }_{i} X_{j_{1}}, X_{j_{2}}\right] X_{j_{1}}^{3-i} f\right)_{0}\right|+\varepsilon\left\|X_{j_{1}}^{2} X_{j_{2}} f\right\|_{0}^{2}+C(\varepsilon) I_{0} \\
& \leqslant\left|\left(X_{j_{2}} X_{j_{1}} X_{j_{2}} f, X_{j_{1}}^{3} f\right)_{0}\right|+C \sum_{j=1}^{k}\left\|X_{j}^{2} f\right\|_{\frac{1}{2}}+\varepsilon\left\|X_{j_{1}}^{2} X_{j_{2}} f\right\|_{0}^{2}+C(\varepsilon) I_{0} \\
& \leqslant \varepsilon \sum_{j=1}^{k}\left\|X_{j}^{2} X_{j_{2}} f\right\|_{0}^{2}+C(\varepsilon) I_{0} .
\end{aligned}
$$

Choosing $\varepsilon=\frac{1}{2 k^{3}}$ and then summing up the inequalities just obtained one gets

$$
T(f) \leqslant \frac{T(f)}{2}+C I_{0}
$$

where $T(f)$ denotes $\max _{j_{1}, j_{2}=\overline{1, k}}\left\{\left\|X_{j_{1}}^{2} X_{j_{2}} f\right\|_{0}^{2}\right\}$, from which the needed estimate in the case $s=0$ follows. To prove the estimate (4.14) in the general case $s \in \mathbb{R}$ we need to substitute $G^{s} f$ for $f$ in the inequality just obtained. We have

$$
\left\|X_{j_{1}}^{2} X_{j_{2}} G^{s} f\right\|_{0}^{2} \leqslant C\left(\sum_{j=1}^{k}\left\|X_{j}^{3} G^{s} f\right\|_{\frac{1}{6}}^{2}+\left\|G^{s} f\right\|_{0}^{2}\right) \leqslant C\left(\sum_{j=1}^{k}\left\|X_{j}^{3} f\right\|_{s+\frac{1}{6}}^{2}+\|f\|_{s}^{2}\right) .
$$

On the other hand,

$$
\begin{aligned}
\left\|X_{j_{1}}^{2} X_{j_{2}} f\right\|_{s}^{2} & \leqslant C\left(\left\|G^{s} X_{j_{1}}^{2} X_{j_{2}} f\right\|_{0}^{2}+\|f\|_{s}^{2}\right) \leqslant C\left(\left\|X_{j_{1}}^{2} X_{j_{2}} G^{s} f\right\|_{0}^{2}+\|f\|_{s}^{2}\right) \\
& \left.\left.+C \sum_{\substack{\iota^{1} \subseteq\left(j_{1}, j_{1}, j_{2}\right),\left|\iota^{1}\right| \leqslant 2\\
}}\|C\| X_{j_{1}}^{2} X_{j_{2}} G^{s} f \|_{0}^{2}+j_{1}, j_{2}\right) \backslash \iota^{1} X, G^{s}\right] X^{\iota^{1}} f \|_{0}^{2}
\end{aligned}
$$

Comparing the inequalities (4.15), (4.16) we arrive at the desired conclusion.

Proposition 4.32. Let the vector fields $\left\{X_{j}\right\}_{j=1}^{k}$ satisfy the condition $(\mathrm{H})_{3}$ in $\Omega$. Then for each compact $K$ in $\Omega$, and any real value $s$ there exists a constant $C(s, K)=C$ such that

$$
\begin{gathered}
\sum_{0 \leqslant|\iota| \leqslant 2}\left\|X^{\iota} f\right\|_{s+\frac{4-|\iota|}{3}}^{2} \leqslant C\left(\left\|P_{4} f\right\|_{s}^{2}+\|f\|_{s}^{2}\right) \\
\sum_{|\iota|=3}\left\|X^{\iota} f\right\|_{s}^{2} \leqslant C\left(\sum_{j=1}^{k}\left\|X_{j}^{3} f\right\|_{s+\frac{1}{6}}^{2}+\|f\|_{s}^{2}\right) \leqslant C\left(\left\|P_{4} f\right\|_{s}^{2}+\|f\|_{s}^{2}\right)
\end{gathered}
$$

for all $f \in C_{0}^{\infty}(K)$.

Proof. The estimate (4.17) can be proved as in Lemma 4.9 with the use of Theorem 2.4 and Lemma 4.2, It remains to prove the estimate (4.18). We first show it for 
the case $s=0$. We calculate the scalar product of $-\left(P_{4} f, X_{1}^{2} f\right)_{0}$. By Lemma 4.31

$$
\begin{aligned}
\left|\sum_{0 \leqslant|\iota| \leqslant 3}\left(a_{\iota} X^{\iota} f, X_{1}^{2} f\right)_{0}\right| & \leqslant \varepsilon \sum_{0 \leqslant|\iota| \leqslant 3}\left\|X^{\iota} f\right\|_{-\frac{1}{6}}^{2}+C(\varepsilon)\left\|X_{1}^{2} f\right\|_{\frac{1}{6}}^{2} \\
& \leqslant \varepsilon \sum_{j=1}^{k}\left\|X_{j}^{3} f\right\|_{0}^{2}+C(\varepsilon)\|f\|_{0}^{2} .
\end{aligned}
$$

We now estimate the principal terms in $-\left(P_{4} f, X_{1}^{2} f\right)_{0}$. We have

$$
-\left(\sum_{j=1}^{4} X_{j}^{4} f, X_{1}^{2} f\right)_{0}=-\left(X_{1}^{4} f, X_{1}^{2} f\right)_{0}-\sum_{j=2}^{k}\left(X_{j}^{4} f, X_{1}^{2} f\right)_{0}:=I_{1}+\sum_{j=2}^{k} I_{j} .
$$

It is easy to check that

$$
I_{1}=\left\|X_{1}^{3} f\right\|_{0}^{2}+J_{1}, \quad\left|J_{1}\right| \leqslant \varepsilon\left\|X_{1}^{3} f\right\|_{0}^{2}+C(\varepsilon)\|f\|_{0}^{2} .
$$

Next we estimate the term $I_{j}$ with $2 \leqslant j \leqslant k$. By Lemma 4.31

$$
I_{j}=-\left(X_{j}^{2} f, X_{j}^{2} X_{1}^{2} f\right)_{0}+A_{j}, \text { where }\left|A_{j}\right| \leqslant \varepsilon \sum_{j=1}^{k}\left\|X_{j}^{3} f\right\|_{0}^{2}+C(\varepsilon)\|f\|_{0}^{2} .
$$

Furthermore,

$$
\begin{gathered}
-\left(X_{j}^{2} f, X_{j}^{2} X_{1}^{2} f\right)_{0}=\left\|X_{1} X_{j}^{2} f\right\|_{0}^{2}+B_{j}+\sum_{\substack{\left|\iota^{1}\right|+\left|\iota^{2}\right| \leqslant 4 \\
\left|\iota^{1}\right| \geq 2}} C\left(\iota^{1}, \iota^{2}\right)\left(X_{j}^{2} f, X_{\iota^{1}} X^{\iota^{2}} f\right)_{0}, \\
\text { where as before }\left|B_{j}\right| \leqslant \varepsilon \sum_{j=1}^{k}\left\|X_{j}^{3} f\right\|_{0}^{2}+C(\varepsilon)\|f\|_{0}^{2} .
\end{gathered}
$$

On the other hand, in view of the estimate (4.17)

$$
\left|\left(X_{j}^{2} f, X_{\iota^{1}} X^{\iota^{2}} f\right)_{0}\right| \leqslant C\left(\left\|P_{4} f\right\|_{-\frac{1}{6}}^{2}+\|f\|_{0}^{2}\right) \text { for }\left|\iota^{2}\right| \leqslant 2 .
$$

Combining all the obtained inequalities we arrive at the needed result in the case $s=0$. In order to obtain the estimate (4.18) in the general case $s \in \mathbb{R}$ we substitute $G^{s} f$ for $f$ in the inequality just obtained for $s=0$. We omit the details.

Combining Theorem 4.30 and Proposition 4.32 we get

Theorem 4.33. Let the vector fields $\left\{X_{j}\right\}_{j=1}^{k}$ satisfy the condition $(\mathrm{H})_{3}$ in $\Omega$. Then $P_{4}$ is a hypoelliptic operator.

Proof. We show that if $s \in \mathbb{R}$ and $P_{4} f \in H_{\text {loc }}^{s}(\Omega)$, then

$$
\begin{gathered}
X^{\iota} f \in H_{\mathrm{loc}}^{s+\frac{4-|\iota|}{3}}(\Omega) \text { for }|\iota| \leqslant 2, X^{\iota} f \in H_{\mathrm{loc}}^{s}(\Omega) \text { for }|\iota|=3, \\
\text { and } X_{j}^{3} f \in H_{\mathrm{loc}}^{s+\frac{1}{6}}(\Omega) \text { for } j=\overline{1, k},
\end{gathered}
$$

from which the theorem follows. The above claim can first be achieved in the case when $f$ has compact support based on the estimates (4.17), (4.18), Proposition 4.32 and the analogous tricks given in the proof of Corollary 4.29, Furthermore, using a sequence of functions $g_{i}(x)$ as in Theorem 4.30 we can get the desired result in the general case. 
Remark 4.34. A theorem analogous to Theorem 4.33 still remains true for the operator of the form

$$
P_{4}^{\prime}=\sum_{j=1}^{k} A_{j}(x, \partial) X_{j}^{4}+\sum_{|\iota| \leqslant 3} B_{\iota}(x, \partial) X^{\iota},
$$

where $\left\{X_{j}\right\}_{j=1}^{k}$ are proper first order p.d.o. with real principal symbols, and $A_{j}(x, \partial), B_{\iota}(x, \partial)$ are proper zero order p.d.o. Furthermore, operators $A_{j}(x, \partial)$ are assumed to be elliptic and positive.

We now go over to the proof of the maximal $\frac{1}{3}$-hypoellipticity and the extendedly maximal hypoellipticity of the operator $P_{4}$. To this end we need the lifting construction from the vector fields $\left\{X_{j}\right\}_{j=1}^{k}$ in $\Omega$ to the vector fields $\left\{\tilde{X}_{j}\right\}_{j=1}^{k}$ in $\tilde{\Omega}$, where the new system is "free up" to the third step and satisfies the condition $(\mathrm{H})_{3}$ in $\tilde{\Omega}$. First we prove a theorem concerning the "homogeneous" operator

$$
\tilde{P}_{4}^{(0)}=\sum_{j=1}^{k} \tilde{X}_{j}^{4}
$$

in $\tilde{\Omega}$. We will adopt the terminologies and definitions from the paper [15].

Lemma 4.35. Let the system of vector fields $\left\{\tilde{X}_{j}\right\}_{j=1}^{k}$ be free up to the third step and satisfy the condition $(\mathrm{H})_{3}$ in $\tilde{\Omega}$, and $\tilde{a} \in C_{0}^{\infty}(\tilde{\Omega})$. There exist operators $\tilde{T}, \tilde{S}, \tilde{S}{ }^{\prime}$ such that

i) $\tilde{T}$ is of type 4 .

ii) $\tilde{S}, \tilde{S}^{\prime}$ are of type 1 .

iii) $\tilde{P}_{4}^{(0)} \tilde{T}=\tilde{a} \tilde{I}+\tilde{S}, \tilde{T} \tilde{P}_{4}^{(0)}=\tilde{a} \tilde{I}+\tilde{S}^{\prime}$, where by $\tilde{I}$ we denote the unit transform.

Proof. By Theorem 5 from [15], the vector fields $\left\{\tilde{X}_{j}\right\}_{j=1}^{k}$ can be approximated by the vector fields $\left\{Y_{j}\right\}_{j=1}^{k}$ on the group $G_{k, 3}$ (recall that the vector fields $\left\{Y_{j}\right\}_{j=1}^{k}$ form a basis of the Lie algebra $\mathfrak{g}_{k, 3}$ and $\left.\operatorname{dim} \tilde{\Omega}=\operatorname{dim} G_{k, 3}\right)$. Consider the operator

$$
\mathfrak{Q}_{4}=\sum_{j=1}^{k} Y_{j}^{4} .
$$

By Theorem 4.33, the operator $\mathfrak{Q}_{4}$ and its formal adjoint are hypoelliptic on $G_{k, 3}$. Therefore by Proposition A in [15], taking into account the fact that $4<\operatorname{dim} G_{k, 3}$ there can be found a fundamental solution $K_{4}(u)$ of the operator $\mathfrak{Q}_{4}$, satisfying the following conditions:

1) Function $K_{4}$ is of type 4 on the group $G_{k, 3}$.

2) The following formula holds: $\mathfrak{Q}_{4} K_{4}(u)=\delta(u)$, where $\delta$ is the Dirac measure on the group $G_{k, 3}$.

Let $\tilde{a} \lesssim \tilde{b}$. Put

$$
\tilde{K}_{4}(\tilde{x}, \tilde{y})=\tilde{a}(\tilde{x}) K_{4}(\Theta(\tilde{x}, \tilde{y})) \tilde{b}(\tilde{y})
$$

and define the operator $\tilde{T}$ by

$$
\tilde{T} \tilde{f}(\tilde{x})=\int_{\tilde{\Omega}} \tilde{K}_{4}(\tilde{x}, \tilde{y}) \tilde{f}(\tilde{y}) \mathrm{d} \tilde{y} .
$$


In order to verify the properties ii) and iii) in Lemma 4.35, note that

$$
\tilde{P}_{4}^{(0)}=\sum_{j=1}^{k} \tilde{X}_{j}^{4}=\sum_{j=1}^{k}\left(Y_{j}+R_{j}\right)^{4}=\sum_{j=1}^{k} Y_{j}^{4}+\Delta,
$$

where $\Delta$ is a differential operator of local degree $\leqslant 3$. Hence,

$$
\Delta \tilde{K}_{4}(\tilde{x}, \tilde{y})=\tilde{K}^{\prime}, \tilde{P}_{4}^{(0)} \tilde{K}_{4}(\tilde{x}, \tilde{y})=\tilde{a}(\tilde{x})\left(\mathfrak{Q}_{4} K_{4}(u)\right) \tilde{b}(\tilde{y})+\tilde{K}^{\prime \prime}=\tilde{a}(\tilde{x}) \delta(\tilde{x}-\tilde{y})+\tilde{K}^{\prime \prime \prime},
$$

where $\tilde{K}^{\prime}, \tilde{K}^{\prime \prime}, \tilde{K}^{\prime \prime \prime}$ are kernels of type 1 . Thus we get the claim for $\tilde{P}_{4}^{(0)} \tilde{T}$. Integrating by parts we can obtain the claim for $\tilde{T} \tilde{P}_{4}^{(0)}$. Lemma 4.35 is proved.

Theorem 4.36. Let the system of vector fields $\left\{\tilde{X}_{j}\right\}_{j=1}^{k}$ be free up to step 3 and satisfy the condition $(\mathrm{H})_{3}$ in $\tilde{\Omega}$, and let there be given functions $\tilde{a}, \tilde{b}, \tilde{c} \in C_{0}^{\infty}(\tilde{\Omega})$ such that $\tilde{a} \lesssim \tilde{b} \lesssim \tilde{c}$. Then for each positive integer $r$ there can be found operators $\tilde{T}_{r}$ and $\tilde{S}_{r}$ such that

$$
\tilde{T}_{r} \tilde{b} \tilde{P}_{4}^{(0)}=\tilde{a} \tilde{I}+\tilde{S}_{r} \cdot \tilde{c}
$$

where $\tilde{T}_{r}$ is a smoothing operator of order 4 , and $\tilde{S}_{r}$ is a smoothing operator of order $r$.

Proof. Let there be given three functions $\tilde{\alpha}, \tilde{\beta}, \tilde{\gamma}$ in $C_{0}^{\infty}(\tilde{\Omega})$ such that $\tilde{\alpha} \lesssim \tilde{\beta} \lesssim \tilde{\gamma}$. We can always find a function $\tilde{\beta}_{0}$ such that $\tilde{\alpha} \lesssim \tilde{\beta}_{0} \lesssim \tilde{\beta}$. Now applying Lemma 4.35 with $\tilde{a}$ replaced by $\tilde{\alpha}$ and $\tilde{f}$ replaced by $\tilde{\beta}_{0} \tilde{f}$ we obtain $\tilde{T}\left(\tilde{P}_{4}^{(0)} \tilde{\beta}_{0}\right)=\tilde{\alpha} \tilde{I}+\tilde{S}^{\prime} \tilde{\beta}_{0}$. However,

$$
\begin{aligned}
\tilde{P}_{4}^{(0)}\left(\tilde{\beta}_{0} \tilde{f}\right) & =\tilde{\beta}_{0} \tilde{P}_{4}^{(0)}(\tilde{f})+\tilde{f} \tilde{P}_{4}^{(0)}\left(\tilde{\beta}_{0}\right) \\
& +\sum_{j=1}^{k}\left(4 \tilde{X}_{j}\left(\tilde{\beta}_{0}\right) \tilde{X}_{j}^{3}(\tilde{f})+6 \tilde{X}_{j}^{2}\left(\tilde{\beta}_{0}\right) \tilde{X}_{j}^{2}(\tilde{f})+4 \tilde{X}_{j}^{3}\left(\tilde{\beta}_{0}\right) \tilde{X}_{j}(\tilde{f})\right) .
\end{aligned}
$$

Now put $\tilde{T}_{1}=\tilde{T} \tilde{\beta}_{0}$ and

$\tilde{S}_{1} \tilde{f}=\tilde{S}^{\prime}\left(\tilde{\beta}_{0} \tilde{f}\right)-\tilde{f} \tilde{P}_{4}^{(0)}\left(\tilde{\beta}_{0}\right)-\sum_{j=1}^{k}\left(4 \tilde{X}_{j}\left(\tilde{\beta}_{0}\right) \tilde{X}_{j}^{3}(\tilde{f})+6 \tilde{X}_{j}^{2}\left(\tilde{\beta}_{0}\right) \tilde{X}_{j}^{2}(\tilde{f})+4 \tilde{X}_{j}^{3}\left(\tilde{\beta}_{0}\right) \tilde{X}_{j}(\tilde{f})\right)$.

It is not difficult to check that $\tilde{S}_{1} \tilde{\gamma} \tilde{f}=\tilde{S}_{1} \tilde{f}$. Thus,

$$
\tilde{T}_{1}\left(\tilde{\beta} \tilde{P}_{4}(\tilde{f})\right)=\tilde{\alpha} \tilde{f}+\tilde{S}_{1}(\tilde{\gamma} \tilde{f}) .
$$

The last equality is the claim of Theorem 4.36 for $r=1$. In order to prove the theorem for general $r$ we need to repeat the arguments used in the proof of Corollary 17.4 in [15] combined with the claim just obtained for $r=1$. We will not elaborate. Theorem 4.36 is proved.

Theorem 4.37. Let the system of vectors fields $\left\{\tilde{X}_{j}\right\}_{j=1}^{k}$ be free up to step 3 and satisfy the condition $(\mathrm{H})_{3}$ in $\tilde{\Omega}$. Then $\tilde{P}_{4}$ is maximally $\frac{1}{3}$-hypoelliptic and extendedly maximally hypoelliptic in the system $\left\{X_{j}\right\}_{j=1}^{k}$ in $\tilde{\Omega}$.

Proof. In view of Remark 2.8 it suffices to establish the maximal $\frac{1}{3}$-hypoellipticity and the extendedly maximal hypoellipticity of $\tilde{P}_{4}^{(0)}$ in the system $\left\{X_{j}\right\}_{j=1}^{k}$. First we prove the maximal $\frac{1}{3}$-hypoellipticity of $\tilde{P}_{4}^{0}$. Note that the estimates for $\tilde{f},\left\{\tilde{X} \tilde{f}_{j}\right\}_{j=1}^{k}$, $\left\{\tilde{X}^{\iota} \tilde{f}\right\}_{|\iota|=2}$ were obtained in Proposition 4.32, It remains to establish the estimates 
for $\left\{\tilde{X}^{\iota} \tilde{f}\right\}_{|\iota|=3},\left\{\tilde{X}^{\iota} \tilde{f}\right\}_{|\iota|=4}$. Let $\tilde{K}$ be a compact in $\tilde{\Omega}$ and $\tilde{a} \in C_{0}^{\infty}(\tilde{\Omega}), \tilde{a} \equiv 1$ on $\tilde{K}$. Applying Theorem 4.36 with the help of Theorems 8 and 12 in [15] we have

$$
\left\|\tilde{X}^{\iota} \tilde{f}\right\|_{s} \leqslant C\left(\left\|\tilde{X}^{\iota} \tilde{T} \tilde{P}_{4}^{(0)} \tilde{f}\right\|_{s}+\|\tilde{f}\|_{s-\frac{1}{3}}\right) .
$$

Since $\tilde{T}$ is of order 4 , by Theorem 8 from [15], operator $\tilde{X}^{\iota} \tilde{T}$ is of order 1 for $|\iota|=3$ and of order 0 for $|\iota|=4$. Therefore

$$
\left\|\tilde{X} \iota \tilde{T} \tilde{P}_{4}^{(0)} \tilde{f}\right\|_{s} \leqslant C\left(\left\|\tilde{P}_{4}^{(0)} \tilde{f}\right\|_{s-\frac{1}{3}}+\|\tilde{f}\|_{s-\frac{1}{3}}\right), \quad|\iota|=3 .
$$

Combining estimates (4.19) and (4.20) we get the desired estimates for $\left\{\tilde{X}^{\iota} \tilde{f}\right\}_{|\iota|=3}$. Similarly, one can prove the estimate for $\left\{\tilde{X}^{\iota} \tilde{f}\right\}_{|\iota|=4}$.

Next we prove the extendedly maximal hypoellipticity of $\tilde{P}_{4}^{(0)}$ in the system $\left\{X_{j}\right\}_{j=1}^{k}$. Let

$$
\tilde{f} \in L_{\mathrm{loc}}^{2}(\tilde{\Omega}), \tilde{P}_{4}^{(0)} \tilde{f} \in S_{\mathrm{loc}}^{m,\left\{\tilde{X}_{j}\right\}_{j=1}^{k}}(\tilde{\Omega}), \tilde{b} \in C_{0}^{\infty}(\tilde{\Omega}) .
$$

Take a function $\tilde{a} \in C_{0}^{\infty}(\tilde{\Omega})$ such that $\tilde{b} \lesssim \tilde{a}$. Using Theorem 4.36 and Theorem 12 from [15] one deduces that

$$
\begin{aligned}
\tilde{b} \tilde{f} & =\tilde{a}(\tilde{b} \tilde{f})=\tilde{T} \tilde{P}_{4}^{(0)}(\tilde{b} \tilde{f})+\tilde{S}_{1}(\tilde{b} \tilde{f})=\tilde{T} \tilde{a} \tilde{P}_{4}^{(0)} \tilde{f} \\
& +\sum_{j=1}^{k} \sum_{i=1}^{4} C_{4}^{i} \tilde{T} \tilde{X}_{j}^{4-i}\left[\tilde{b},{ }_{i} \tilde{X}_{j}\right](\tilde{b} \tilde{f})+\tilde{S}_{1}(\tilde{b} \tilde{f}) \in S_{\operatorname{loc}}^{1,\left\{\tilde{X}_{j}\right\}_{j=1}^{k}(\tilde{\Omega}) .}
\end{aligned}
$$

In view of the arbitrariness of $\tilde{b}$ we conclude that $\tilde{f} \in S_{\mathrm{loc}}^{1,\left\{\tilde{X}_{j}\right\}_{j=1}^{k}}(\tilde{\Omega})$. From the statement just obtained we have

$$
\begin{aligned}
\tilde{S}_{1}(\tilde{b} \tilde{f}) \in S_{\mathrm{loc}}^{2,\left\{\tilde{X}_{j}\right\}_{j=1}^{k}}(\tilde{\Omega}), \tilde{T} \tilde{a} \tilde{P}_{4}^{(0)} \tilde{f} & \in S_{\mathrm{loc}}^{m+4,\left\{\tilde{X}_{j}\right\}_{j=1}^{k}}(\tilde{\Omega}), \\
\tilde{T} \tilde{X}_{j}^{4-i}\left[\tilde{b},{ }_{i} \tilde{X}_{j}\right](\tilde{b} \tilde{f}) & \in S_{\mathrm{loc}}^{1+i,\left\{\tilde{X}_{j}\right\}_{j=1}^{k}}(\tilde{\Omega}), i=1, \ldots, 4,
\end{aligned}
$$

from which, in view of arbitrariness of $\tilde{b}$, it follows that $\tilde{f} \in S_{\text {loc }}^{2,\left\{\tilde{X}_{j}\right\}_{j=1}^{k}}(\tilde{\Omega})$. Iterating the procedure $m+2$ times, we arrive at the desired result.

We now turn back to the original domain $\Omega$. We can define the extension and restriction operators $E, R$ as in 15 . To a given operator $\tilde{T}$ mapping functions on $\tilde{\Omega}$ into functions on $\tilde{\Omega}$ we associate with its restriction on $\Omega$ by the formula: $T=R \tilde{T} E$.

Theorem 4.38. Let the vector fields $\left\{X_{j}\right\}_{j=1}^{k}$ satisfy the condition $(\mathrm{H})_{3}$ in $\Omega$, and let $a \in C_{0}^{\infty}(\Omega)$. Then there exist operators $T$ (parametrix) and $S, S^{\prime}$ such that

i) $T$ is smoothing of order 4 .

ii) $S, S^{\prime}$ are smoothing of order 1 .

iii) $P_{4} T=a I+S, T P_{4}=a I+S^{\prime}$, where by $I$ we denote the unit transform.

Proof. It is clear that $T$ is a parametrix for $P_{4}$ if and only if $T$ is a parametrix for $P_{4}^{(0)}$. Hence, it suffices to prove Theorem 4.38 for $P_{4}^{(0)}$. Let $\varphi \lesssim \psi$. Put $\tilde{a}(x, t)=a(x) \psi(t)$. By Lemma 4.35 there exist operators $\tilde{T}, \tilde{S}, \tilde{S}^{\prime}$ of type 4 and 1 , respectively, such that

$$
\tilde{P}_{4}^{(0)} \tilde{T}=\tilde{a} \tilde{I}+\tilde{S} \text {, and } \tilde{T} \tilde{P}_{4}^{(0)}=\tilde{a} \tilde{I}+\tilde{S}^{\prime} .
$$


Multiply the second equation in (4.21) by $R$ on the left and by $E$ on the right. Obviously, $R \tilde{a} \tilde{I} E=a I$. Let $S^{\prime}=R \tilde{S}^{\prime} E$ and $T=R \tilde{T} E$. Taking into account the fact that

$$
\tilde{X}_{j_{1}} \tilde{X}_{j_{2}} \cdots \tilde{X}_{j_{l}} E=E X_{j_{1}} X_{j_{2}} \cdots X_{j_{l}}
$$

we deduce that $\tilde{P}_{4}^{(0)} E=E P_{4}^{(0)}$. Combining these we get $T P_{4}^{(0)}=a I+S^{\prime}$.

Now we go over to the proof of the equality for $P_{4}^{(0)} T$. Note that

$$
P_{4}^{(0)} T=P_{4}^{(0)} R \tilde{T} E=R P_{4}^{(0)} \tilde{T} E+\left[P_{4}^{(0)}, R\right] \tilde{T} E .
$$

However, in view of formula (17.10) in [15]

$$
P_{4}^{(0)} R-R \tilde{P}_{4}^{(0)}=\sum_{0<|\iota| \leqslant 3} R_{\iota}^{\prime} \tilde{X}^{\iota}+R_{0}^{\prime}
$$

Hence

$$
P_{4}^{(0)} T=a I+R \tilde{S} E+\sum_{0<|\iota| \leqslant 3} R_{\iota}^{\prime} \tilde{X} \iota \tilde{T} E+R_{0}^{\prime} \tilde{T} E .
$$

Thus, we can take $S=R \tilde{S} E+\sum_{0<|\iota| \leqslant 3} R_{\iota}^{\prime} \tilde{X}_{\iota} \tilde{T} E+R_{0}^{\prime} \tilde{T} E$ and the property of $S$ follows from the property of $R_{\iota}^{\prime}, R_{0}^{\prime}, E$. Theorem 4.38 is proved.

Proposition 4.39. Let the vector fields $\left\{X_{j}\right\}_{j=1}^{k}$ satisfy the condition $(\mathrm{H})_{3}$ in $\Omega$, and let there be given functions $a, b, c \in C_{0}^{\infty}(\Omega)$ such that $a \lesssim b \lesssim c$. Then for each integer $r$ there exist operators $T_{r}$ and $S_{r}$ such that

$$
T_{r} b P_{4}=a I+S_{r} \cdot c,
$$

where $T_{r}$ is smoothing of order 4 , and $S_{r}$ is smoothing of order $r$.

Proof. Based on Theorem 4.38 we can prove the theorem for $P_{4}^{(0)}$ along the lines of the proof of Theorem 4.36. However, if Proposition 4.39 is true for $P_{4}^{(0)}$, then it is easily seen that it is true for $P_{4}$. We omit the details.

In the following theorem we use the spaces $L_{\alpha, \text { loc }}^{p}, \Lambda_{\alpha, \text { loc }}$, definitions of which can be found in the book [16].

Theorem 4.40. Let the vector fields $\left\{X_{j}\right\}_{j=1}^{k}$ satisfy the condition $(\mathrm{H})_{3}$ in $\Omega$. Suppose that $f \in L_{\mathrm{loc}}^{p}(\Omega), p \in(1, \infty)$ and $P_{4} f=g$. Then

i) If $g \in L_{\alpha, \text { loc }}^{p}(\Omega), \alpha \geq 0$, then $f \in L_{\alpha+\frac{4}{3}, \text { loc }}^{p}(\Omega)$.

ii) If $g \in \Lambda_{\alpha, \text { loc }}(\Omega), \alpha>0$, then $f \in \Lambda_{\alpha+\frac{4}{3}, \text { loc }}(\Omega)$.

iii) If $g \in L^{\infty}(\Omega)$, then $f \in \Lambda_{\frac{4}{3}, \text { loc }}(\Omega)$.

iv) If $g \in S_{p, \text { loc }}^{m,\left\{X_{j}\right\}_{j=1}^{k}}(\Omega), p \in(1, \infty), k=0,1, \ldots$, then $f \in S_{p, \text { loc }}^{m+4,\left\{X_{j}\right\}_{j=1}^{k}}(\Omega)$.

Proof. By applying the adjoint formula of (4.22) the proof then follows the lines of the proof of Theorem 16 in [15] with some obvious modifications. We leave the details for the readers. Theorem 4.40 is proved.

Theorem 4.41. Let the vector fields $\left\{X_{j}\right\}_{j=1}^{k}$ satisfy the condition $(\mathrm{H})_{3}$ in $\Omega$. If the function $\Phi\left(x, \tau_{\iota}\right)_{|\iota| \leqslant 3}$ is infinitely differentiable, then $\Psi_{4}$ is a nonlinear hypoelliptic 
operator. Moreover, it is extendedly maximally hypoelliptic in the system $\left\{X_{j}\right\}_{j=1}^{k}$ in $\Omega$.

Proof. In view of Theorem4.33. by the same argument used in the proof of Theorem 4.13 one can state that if a function $f$ is a solution of the equation $\Psi_{4}\left(x, \partial^{\alpha}\right) f=$ $g \in C^{\infty}(\Omega)$ and $f \in C^{3(n+1)}(\Omega)$, then $f \in C^{\infty}(\Omega)$.

Example 4.42. The nonlinear operator

$$
\frac{\partial^{4}}{\partial x^{4}}+\frac{\partial^{4}}{\partial y^{4}}+x^{4} y^{4} \frac{\partial^{4}}{\partial z^{4}}+\mathrm{e}^{x^{2} y^{2} \frac{\partial^{2}}{\partial z^{2}} \frac{\partial}{\partial x}}
$$

is hypoelliptic in $\mathbb{R}^{3}$.

\section{ACKNOWLEDGMENTS}

The author of this paper would like to thank Professor B. Helffer for some valuable remarks which improved the paper.

\section{REFERENCES}

1. S. Bernstein, Sur la nature analytique des solutions des équations aux dériveés partielles du second ordre, Math. Ann., 59 (1904), 20-76. MR1511259

2. A. Douglis, L. Nirenberg, Interior estimates for elliptic systems of partial differential equations, Comm. Pure Appl. Math., 8 (1955), 503-538. MR0075417 (17:743b)

3. Yu. V. Egorov, Linear Differential Equations of Principal Type, Moscow, Nauka, 1984, 360. MR.776969 (86f:35001)

4. Yu. V. Egorov, N. M. Tri, Maximally hypoelliptic operators with a noninvolutive characteristic set, DAN SSSR., 314 (1990), 1059-1061. MR.1116867 (92i:35034)

5. Yu. V. Egorov, N. M. Tri, On a class of maximally hypoelliptic operators, Transactions Sem. Petrovskii, 17 (1994), 3-26. MR1373419

6. G. B. Folland, Applications of analysis on nilpotent groups to partial differential equations, Bull. Amer. Math. Soc., 83 (1977), 912-930. MR0457928(56:16132)

7. A. Friedman, On classes of solutions of elliptic linear partial differential equations, Proc. Amer. Math. Soc., 8 (1957), 418-427. MR0096894 (20:3376)

8. B. Helffer, J. Nourrigat, Hypoellipticite Maximal pour des Operateurs Polynomes de Champ de Vecteur, Birkhauser (1985), 275 p. MR897103 (88i:35029)

9. L. Hörmander, The Analysis of Linear Partial Differential Operators, III, Springer-Verlag, Berlin, Heidelberg, 1985. MR781536 (87d:35002a)

10. L. Hörmander, Hypoelliptic second-order differential operators, Acta Math., 119 (1967), 147171. MR0222474 (36:5526)

11. J. J. Kohn, Pseudo-differential operators and hypo-ellipticity, Proc. Symp. Pure Math., 23 (1973), 61-69. MR0338592 (49:3356)

12. J. J. Kohn, Hypoellipticity and loss of derivatives, Ann. of Math. (2) 162 (2005), 943-986. MR 2183286 (2006k:35036)

13. C. Miranda, Partial Differential Equations of Elliptic Type, Springer-Verlag, 1970, 370. MR 0284700 (44:1924)

14. I. G. Petrovskii, Sur l'analyticite des solutions des systèmes d'équations différentielles, Matem. Sbornik (N. S.), 5(47) (1939), 3-70.

15. L. P. Rothschild, E. M. Stein, Hypoelliptic differential operators and nilpotent groups, Acta Math., 137 (1976), 247-320. MR0436223 (55:9171)

16. E. M. Stein, Singular Integrals and Differentiability Properties of Functions, Princeton, New Jersey: Princeton University Press, 1970. MR0290095 (44:7280)

17. M. Taylor, Pseudodifferential Operators, Princeton: Princeton University Press, 1981. MR $618463(82 \mathrm{i}: 35172)$

18. F. Treves, Introduction to Pseudo-Differential Operators and Fourier Integral Operators, Plenum Press, 1982. MR597145 (82i:58068) 
19. V. T. T. Hien, N. M. Tri, Analyticity of solutions of semi-linear equations with double characteristics, J. Math. Anal. Appl., 337 (2008), 1249-1260.

20. C. J. Xu, Regularity for quasilinear second-order subelliptic equations, Comm. Pure Appl. Math., 45 (1992), 77-96. MR1135924 (93b:35042)

Institute of Mathematics, 18 Hoang Quoc Viet Road, Cau Giay District, 10307 Hanoi, VIETNAM

E-mail address: triminh@math.ac.vn

Current address: Department of Mathematics, University of Chicago, 5734 S. University Avenue, Chicago, Illinois 60637 